\title{
Association between Abnormal Fetal Head Growth and Autism Spectrum
}

\section{Disorder}

Ohad Regev ${ }^{1,2}$, MD, PhD Candidate; Gal Cohen ${ }^{1}$, MD Candidate; Amnon Hadar ${ }^{3,4}$, MD;

Jenny Schuster ${ }^{3}$, BA, MBA; Hagit Flusser ${ }^{5,6}$, MD; Analya Michaelovski ${ }^{5,6}$, MD; Gal Meiri ${ }^{5,7}$, MD;

Ilan Dinstein ${ }^{2,6,8}, \mathrm{PhD}$; Reli Hershkovitch ${ }^{4}, \mathrm{MD}$; and Idan Menashe ${ }^{1,2,6}, \mathrm{PhD}$.

${ }^{1}$ Department of Public Health, Ben-Gurion University of the Negev, Beer-Sheva, Israel;

${ }^{2}$ Zlotowski Center for Neuroscience, Ben-Gurion University of the Negev, Beer-Sheva, Israel;

${ }^{3}$ Clalit Health Services, Beer-Sheva, Israel;

${ }^{4}$ Division of Obstetrics and Gynecology, Soroka University Medical Center, Beer-Sheva, Israel;

${ }^{5}$ Child Development Center, Soroka University Medical Center, Beer-Sheva, Israel;

${ }^{6}$ National Autism Research Center of Israel, Ben-Gurion University of the Negev, Beer-Sheva,

Israel; ${ }^{7}$ Preschool Psychiatric Unit, Soroka University Medical Center, Beer-Sheva, Israel;

${ }^{8}$ Department of Psychology, Ben-Gurion University of the Negev, Beer-Sheva, Israel.

Corresponding Author:

Idan Menashe, $\mathrm{PhD}$

Department of Public Health, Faculty of Health Sciences,

Ben-Gurion University of the Negev, Beer-Sheva 8410501, Israel

Phone: +972-8-6477456

Email: idanmen@bgu.ac.il

Keywords: Autism Spectrum Disorder, Prenatal Ultrasound, Head Growth, Fetal

develonment 
medRxiv preprint doi: https://doi.org/10.1101/2020.08.09.20170811; this version posted August 23, 2020. The copyright holder for this preprint (which was not certified by peer review) is the author/funder, who has granted medRxiv a license to display the preprint in All rights reserved. No reuse allowed without permission.

\section{$1 \quad$ Abstract}

2 Despite evidence for prenatal onset of abnormal head growth in children with autism spectrum disorder (ASD),

3 fetal ultrasound studies in ASD are limited and controversial. We conducted a longitudinal matched case4 sibling-control study on fetal ultrasound biometric measures from 174 ASD children, their own typically 5 developed siblings (TDS; $\mathrm{n}=178$ ) and other population-based typically developed children (TDP; $\mathrm{n}=176$ ).

6 During second trimester, ASD and TDS fetuses had significantly smaller biparietal diameter (BPD) than TDP 7 fetuses $\left(\mathrm{aOR}_{\mathrm{zBPD}}=0.685,95 \% \mathrm{CI}=0.527-0.890\right.$ and $\mathrm{aOR}_{\mathrm{zBPD}}=0.587,95 \% \mathrm{CI}=0.459-0.751$, respectively $)$. 8 Interestingly, sex had a significant effect on head growth with males having larger heads than females within 9 and across groups. Also, males and females with ASD showed different head shapes which were inversely 10 correlated with ASD severity across different gestation periods. Our findings suggest that abnormal fetal head growth is a familial trait of ASD, which is modulated by sex and is associated with the severity of the disorder. 
medRxiv preprint doi: https://doi.org/10.1101/2020.08.09.20170811; this version posted August 23, 2020. The copyright holder for this preprint (which was not certified by peer review) is the author/funder, who has granted medRxiv a license to display the preprint in

All rights reserved. No reuse allowed without permission.

\section{Introduction}

2 Autism spectrum disorder (ASD) is a life-long neurodevelopmental disorder with a complex etiology having

3 both genetic and environmental determinants. ${ }^{1,2}$ The wide range of pre- and perinatal risk factors, ${ }^{3,4}$ together

4 with the abnormalities in early brain development associated with ASD, ${ }^{5-10}$ suggest that, at least in some cases, the predisposition to ASD begins during gestation.

Abnormal head growth has been proposed as an early biomarker for ASD. Specifically, various MRI, postmortem, and head measurement studies have reported that infants who later developed ASD were born with a smaller or average head circumference (HC), followed by an excessive increase during the first years of life in head size, in extra-axial cerebrospinal fluid (CSF) volume, and in brain regions associated with the language, social, emotional and communication functions affected by ASD. ${ }^{7,8,19,20,11-18}$ These studies also reported correlations between clinical severity to postnatal head size, brain morphometry and extra-axial CSF volume. ${ }^{15,19,21,22}$ Importantly, some of these head growth abnormalities have been associated with sex, which is significantly associated with ASD, with the disorder being 4 times more prevalent among males, but symptoms tending to be more frequent and severe among women. ${ }^{23-27}$ Several postnatal studies have found sexspecific neuroanatomical profiles in ASD children compared to sex-matched controls. Among these investigations, a large population-based study revealed sex-specific differences in $\mathrm{HC}$ at birth and throughout early childhood in children with ASD. ${ }^{18}$ Similarly, MRI studies found sex-specific differences in total brain volume (in gray and white matter volume of the frontal and temporal lobes, cingulate cortex, amygdala, and cerebellum), alongside different and abnormal growth trajectories throughout childhood. ${ }^{14,16,22,28-30}$ These studies also showed sex-specific correlations between specific brain regions and the clinical severity of ASD. ${ }^{22}$

Previous postnatal studies suggested that the accelerated head growth among ASD children during early childhood may be a reaction or an adaptation to abnormal prenatal head growth. This assertion is supported by an emerging ASD prenatal literature covering developmental abnormalities in certain fetal ultrasound (US) parameters, ${ }^{31,32}$ and specifically in biometric measures ${ }^{33-37}$ Nevertheless, these studies showed conflicting results. Two studies found indications for either wider heads with normal HC in ASD children compared to 
medRxiv preprint doi: https://doi.org/10.1101/2020.08.09.20170811; this version posted August 23, 2020. The copyright holder for this preprint (which was not certified by peer review) is the author/funder, who has granted medRxiv a license to display the preprint in

All rights reserved. No reuse allowed without permission.

typically developed population (TDP) controls $^{33}$ or larger HC with normal shape compared to population norm. ${ }^{34}$ Two other studies found an association between smaller HC to either ASD phenotype ${ }^{36}$ or autistic traits ${ }^{37}$ while the fifth study didn't find any association between HC and ASD ${ }^{35}$ In addition, the reported studies were conducted on relatively small cohorts and did not account for potential confounders, e.g., none took into consideration familial factors known to play a significant role in fetal growth ${ }^{38}$ and/or to constitute a risk for ASD. ${ }^{39}$ Similarly, several studies did not take into account the inherent male sex bias in ASD,${ }^{33,34}$ even though boys are known to have larger biometric measures. ${ }^{40}$ Finally, these studies report findings from different gestational periods, which further complicates comparing their findings. Thus, conclusions regarding fetal head growth in children with ASD remain minimal, inconsistent and controversial. The main goal of this study, was therefore, to assess fetal head growth in fetuses later diagnosed with ASD in comparison to their typically developed siblings and to a population control across different gestation periods. In addition, we asked whether differences in fetal head growth are modulated by the sex of the fetus and whether they are associated with the clinical severity of children with ASD.

\section{Methods}

\section{Study Sample}

The participants in this case/control study were singletons whose families were members of Clalit Health Services (CHS), Israel's largest health maintenance organization, serving about $75 \%$ of the approximately 700,000 residents of southern Israel, composed of Jews and Bedouins, two ethnic groups that differ in their genetic background and environmental exposures. Members of CHS in this region receive most of their hospitalrelated services (including ASD diagnosis) at the Soroka University Medical Center (SUMC), the region's only tertiary hospital. The diagnosis of ASD at SUMC is a multidisciplinary process, which entails a comprehensive intake interview (clinical and socio-demographic factors), a behavioral evaluation with ADOS-2, ${ }^{41}$ and a full neurocognitive assessment (e.g., Bayley-III ${ }^{42}$ or WPPSI-III ${ }^{43}$ ). Final diagnoses of ASD is made by a pediatric psychiatrist or neurologist, according to DSM-5 criteria. ${ }^{26}$ All sociodemographic and clinical data on these children are stored in the database of the National Autism Research Center of Israel (NARC). ${ }^{44}$ 
medRxiv preprint doi: https://doi.org/10.1101/2020.08.09.20170811; this version posted August 23, 2020. The copyright holder for this preprint (which was not certified by peer review) is the author/funder, who has granted medRxiv a license to display the preprint in

All rights reserved. No reuse allowed without permission.

1 A flowchart showing the case/control assignment for this study is presented in Figure 1. We randomly

2 selected from the NARC database 386 of the 719 children diagnosed with ASD (53.7\%). Among these 386

3 cases, we identified those for whom there were prenatal US scans in the CHS database. The 212 children

4 (54.9\%) for whom US scans were missing were excluded from the study, leaving us with a sample of 174 cases,

5 born between 2008-2017. There were no significant sociodemographic or clinical differences between this

6 sample and other children with ASD in the NARC database, except that the proportion of Jews (vs. Bedouin

7 Israelis) was slightly lower for children included in the study, as was the parental age (Table 1).

8 Of the 174 cases included in the study, 85 had US scans from both the second and third trimesters, while 51 and

938 had scans from only the second or the third trimester, respectively. For each trimester, we matched two types

10 of control group for cases: (1) typically developing siblings (TDS) - for each ASD case, we matched her/his own

11 typically developed sibling who was closest in age; and (2) typically developed population (TDP) - for each case, we identified a typically-developed child matched by year of birth, sex and ethnicity. In total, the study sample included 528 children: 174 with ASD, 178 TDS, and 176 TDP (Figure 1).

\begin{tabular}{llll}
\hline Table 1. Clinical and Sociodemographic Characteristics of ASD Children & \\
\hline Variable & $\begin{array}{l}\text { Included } \\
(\mathbf{n}=\mathbf{1 7 4})\end{array}$ & $\begin{array}{l}\text { Excluded } \\
(\mathbf{n}=\mathbf{5 4 5})\end{array}$ & P-Value \\
\hline Jewish, no. $(\%)^{\mathrm{a}}$ & $124(72.1)$ & $427(79.8)$ & $\mathbf{0 . 0 3 4}$ \\
\hline Male, no. $(\%)^{\mathrm{a}}$ & $142(82.1)$ & $418(76.7)$ & 0.136 \\
\hline${\text { Moderate salary or lower, No. }(\%)^{\mathrm{a}}}^{\mathrm{a}}$ & $30(61)$ & $104(63.8)$ & 0.743 \\
\hline Special education setting, No. (\%) ${ }^{\mathrm{a}}$ & $47(42)$ & $136(43)$ & 0.844 \\
\hline Mother's age, mean (SD), y $^{\mathrm{b}}$ & $29.3(5.4)$ & $31.5(6.2)$ & $<\mathbf{0 . 0 0 1}$ \\
\hline Father's age, mean (SD), y $^{\mathrm{b}}$ & $32.6(7.4)$ & $34.7(7.6)$ & $\mathbf{0 . 0 0 5}$ \\
\hline Diagnosis age, mean (SD), y $^{\mathrm{b}}$ & $3.1(1.2)$ & $3.2(1.5)$ & 0.639 \\
\hline Cognitive score (IQ), mean (SD) ${ }^{\mathrm{b}}$ & $78.4(13.3)$ & $75.5(17.2)$ & 0.112 \\
\hline ADOS Comparison Score, median $(\mathrm{IQR})^{\mathrm{c}}$ & $8(6-9)$ & $7(6-9)$ & 0.500 \\
\hline
\end{tabular}


medRxiv preprint doi: https://doi.org/10.1101/2020.08.09.20170811; this version posted August 23, 2020. The copyright holder for this preprint (which was not certified by peer review) is the author/funder, who has granted medRxiv a license to display the preprint in

All rights reserved. No reuse allowed without permission.

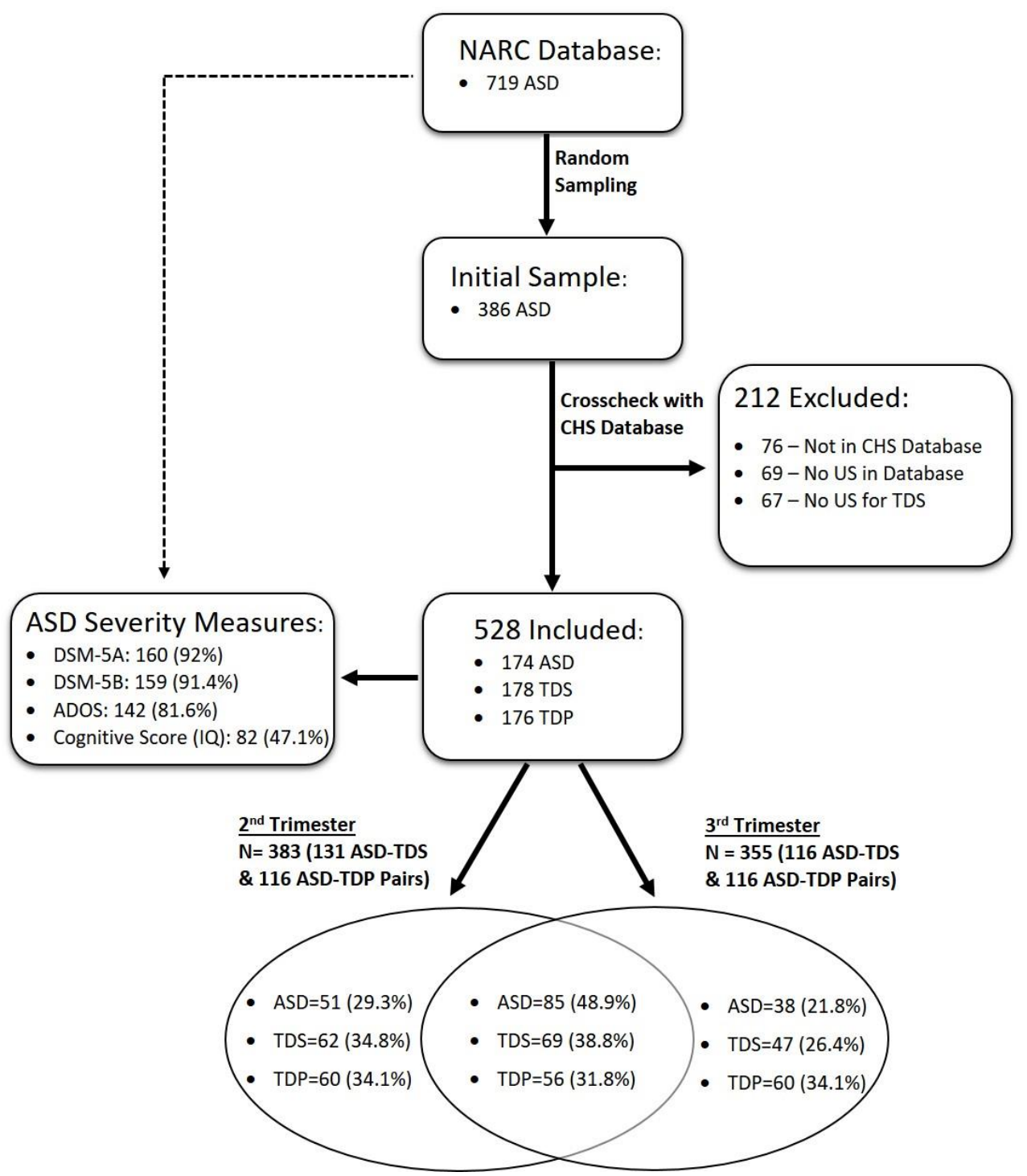

Figure 1. Flowchart of Children included in this Study

$14 \quad$ Fetal Ultrasound Data

15 All the US scans had been performed by experienced sonographers or physicians that recorded fetal biometric measures using standardized US measurements of simple anatomic landmarks to the nearest millimeter. The following biometric measures were extracted from the CHS database for each trimester: HC and head width

18 (BPD, biparietal diameter), i.e., two biometric measures that strongly correlate with brain size; ${ }^{45,46}$ abdominal 19 circumference (AC); and femur length (FL). HC was measured at the level of the BPD, taken at the widest point 20 of the skull at the level of the thalamic peduncles and the cavum septum pellucidum; BPD was measured from 21 outer skull wall to the inner wall directly opposite; AC was measured at the level of the junction of the vertebra, 
medRxiv preprint doi: https://doi.org/10.1101/2020.08.09.20170811; this version posted August 23, 2020. The copyright holder for this preprint (which was not certified by peer review) is the author/funder, who has granted medRxiv a license to display the preprint in

All rights reserved. No reuse allowed without permission.

1 portal sinus, and fetal stomach; and FL was measured from the greater trochanter to the distal metaphysic. ${ }^{33,47,48}$

2 It is known that for these US measurements there is very high inter-rater reliability between experienced

3 sonographers, particularly when transformation to standardized Z-scores are used. ${ }^{33,36,49,50}$ Gestational age (GA)

4 of each child was calculated from the last menstrual period (LMP) and confirmed by the crown-rump length

5 (CRL) from the first trimester. If the date of LMP was unknown, GA was calculated based on CRL.

$6 \quad$ Statistical Analysis

7 We converted all biometric fetal measures to gestation-matched standardized Z-scores using the Hadlock 8 approach, ${ }^{48,51-53}$ the most widely used standardization approach in this field. In addition, we created five new 9 variables by subtracting body parameter (AC and FL) Z-scores from head parameter (HC and BPD) Z-scores 10 and by subtracting the HC Z-scores from the BPD Z-scores to assess fetal head growth relative to the other 11 biometric measures..$^{33,35}$ If there was more than one US measure for a fetus in a particular trimester, we used the 12 mean of its Z-scores as a representative measure. Notably, biometric measures with Z-scores $> \pm 6$ or that 13 deviated by $>3$ SD from the average for that trimester were considered outliers and removed from further 14 analyses (29 outliers: 12 ASD, 9 TDP, 8 TDS).

15 Differences in sociodemographic characteristics between cases and the two control groups were assessed by using the appropriate univariate statistics. Differences in biometric measures between cases and each of the two matched control groups (TDS and TDP) were assessed using paired t-tests and multivariable conditional regression adjusting for sex and maternal age. Differences in biometric measures between the two matched control groups were assessed via unpaired t-tests and multivariable unconditional logistic regression adjusting for sex, maternal age and ethnicity. For fetuses with longitudinal data, a linear mixed-effect model was used to assess the association between fetal growth rate (measured as the difference in biometric measures throughout pregnancy) and ASD, taking into account repeated biometric measures, ethnicity and gestational age. ${ }^{36}$ These analyses were conducted separately for males and females to examine the effect of sex on fetal growth rate.

24 Lastly, correlation coefficients were calculated in cases between biometric measures and ASD severity according to ADOS-2, DSM-5 and cognitive measures. P-values of analyses with multiple testing were adjusted 
medRxiv preprint doi: https://doi.org/10.1101/2020.08.09.20170811; this version posted August 23, 2020. The copyright holder for this preprint (which was not certified by peer review) is the author/funder, who has granted medRxiv a license to display the preprint in perpetuity.

All rights reserved. No reuse allowed without permission.

using the Bonferroni correction. All analyses were conducted using SPSS Statistics V. 25 and R software. A two-sided test significance level of 0.05 was used throughout the entire study.

\section{Results}

5 Clinical and sociodemographic characteristics of the study sample are shown in Table 2. As expected, the sex ratio for the TDS group was significantly different from that for the ASD group, close to 1:1 vs 4:1 male/female, respectively ( $\mathrm{p}<0.001$ ). In addition, for the TDP group, US scans were performed earlier in the second trimester compared to cases $(21.4 \pm 2.8$ vs. $22.3 \pm 1.8$ weeks, respectively; $\mathrm{p}=0.006)$. No other statistically significant

9 differences were seen between cases and controls.

Table 2. Clinical and Sociodemographic Characteristics for Children included in this Study

\begin{tabular}{|c|c|c|c|c|}
\hline Variable & & $\operatorname{ASD}(n=174)$ & TDS $(n=178)$ & $\operatorname{TDP}(n=176)$ \\
\hline Ethnicity (Jewish) & $\begin{array}{l}\text { No. }(\%) \\
\text { P-value }\end{array}$ & $127(73)$ & $\begin{array}{l}131(73.6) \\
1\end{array}$ & $\begin{array}{l}139(79) \\
0.380\end{array}$ \\
\hline Sex (male) & $\begin{array}{l}\text { No. (\%) } \\
\text { P-value }^{\mathrm{a}}\end{array}$ & $140(80.5)$ & $\begin{array}{l}98(55.1) \\
<0001\end{array}$ & $146(83)$ \\
\hline Pregnancy number & $\begin{array}{l}\text { Median (IQR) } \\
\text { P-value }\end{array}$ & $2(1-3)$ & $\begin{array}{l}2(2-4) \\
0.208\end{array}$ & $\begin{array}{l}2(1-3) \\
0.172\end{array}$ \\
\hline Previous abortions & $\begin{array}{l}\text { Median (IQR) } \\
\text { P-value }^{\mathrm{d}}\end{array}$ & $0(0-1)$ & $\begin{array}{l}0(0-1) \\
1\end{array}$ & $\begin{array}{l}0(0-1) \\
0.312\end{array}$ \\
\hline C section & $\begin{array}{l}\text { No. }(\%) \\
\text { P-value }\end{array}$ & $31(18.5)$ & $\begin{array}{l}23(13.4) \\
0.400\end{array}$ & $\begin{array}{l}26(17.9) \\
1\end{array}$ \\
\hline Age at birth (weeks) & $\begin{array}{l}\text { Mean (SD) } \\
\text { P-value }^{\mathrm{c}}\end{array}$ & $38.8(2.5)$ & $\begin{array}{l}39.0(2) \\
1\end{array}$ & $\begin{array}{l}39.3(1.4) \\
0.164\end{array}$ \\
\hline Birth weight (g) & $\begin{array}{l}\text { Mean (SD) } \\
\text { P-value }^{c}\end{array}$ & $3174(579)$ & $\begin{array}{l}3186(552) \\
1\end{array}$ & $\begin{array}{l}3324(523) \\
0.052\end{array}$ \\
\hline 1-min low APGAR score $(<7)$ & $\begin{array}{l}\text { No. }(\%) \\
\text { P-value }\end{array}$ & $4(5)$ & $\begin{array}{l}5(5) \\
1\end{array}$ & $\begin{array}{l}6(6) \\
1\end{array}$ \\
\hline Mother's age (years) & $\begin{array}{l}\text { Mean (SD) } \\
\text { P-value }^{\mathrm{c}}\end{array}$ & $28.6(5.8)$ & $\begin{array}{l}28.6(5.2) \\
1\end{array}$ & $\begin{array}{l}29.2(4.8) \\
0.510\end{array}$ \\
\hline Examined by physician & $\begin{array}{l}\text { No. }(\%) \\
\text { P-value }\end{array}$ & $136(85)$ & $\begin{array}{l}140(78.7) \\
0.264\end{array}$ & $\begin{array}{l}90(79.6) \\
0.496\end{array}$ \\
\hline Breech Presentation at US & $\begin{array}{l}\text { No. }(\%) \\
\text { P-value }\end{array}$ & $51(30.9)$ & $\begin{array}{l}39(22.4) \\
0.334\end{array}$ & $\begin{array}{l}40(24.1) \\
0.308\end{array}$ \\
\hline $\begin{array}{l}\text { Gestation age assessed by last menstrual } \\
\text { period }\end{array}$ & $\begin{array}{l}\text { No. }(\%) \\
\text { P-value }\end{array}$ & $115(74.7)$ & $\begin{array}{l}129(75.9) \\
1\end{array}$ & $\begin{array}{l}85(79.4) \\
0.742\end{array}$ \\
\hline Gestational age at $2^{\text {nd }}$ trimester US (weeks) & $\begin{array}{l}\text { Mean (SD) } \\
\text { P-value }^{\mathrm{c}}\end{array}$ & $22.3(1.8)$ & $\begin{array}{l}22.5(1.5) \\
0.632\end{array}$ & $\begin{array}{l}21.4(2.8) \\
\mathbf{0 . 0 0 6}\end{array}$ \\
\hline Gestational age at $3^{\text {rd }}$ trimester US (weeks) & $\begin{array}{l}\text { Mean (SD) } \\
\text { P-value }^{c}\end{array}$ & $32.5(2.6)$ & $\begin{array}{l}32.6(2.4) \\
1\end{array}$ & $\begin{array}{l}33.2(2.9) \\
0.132\end{array}$ \\
\hline
\end{tabular}

$10 \quad{ }^{\mathrm{a}}$ Chi-square; ${ }^{\mathrm{b}}$ Fisher's Exact Test; ${ }^{\mathrm{c}}$ Two-sided t-test; ${ }^{\mathrm{d}}$ Mann-Whitney U test .

11 All p-values are Bonferroni corrected for multiple comparison $(n=2)$. 
medRxiv preprint doi: https://doi.org/10.1101/2020.08.09.20170811; this version posted August 23, 2020. The copyright holder for this preprint (which was not certified by peer review) is the author/funder, who has granted medRxiv a license to display the preprint in

All rights reserved. No reuse allowed without permission.

\section{$\underline{\text { Biometric Measures }}$}

Differences in fetal US biometric measures between cases and controls are shown in Table $\mathbf{3}$ and Figures $\mathbf{2 \& 3}$. In the second trimester, both ASD and TDS fetuses had significantly narrower heads (smaller BPD) than TDP fetuses $\left(\mathrm{aOR}_{\mathrm{zBPD}}=0.685,95 \% \mathrm{CI}=0.527-0.890\right.$ and $\mathrm{aOR}_{\mathrm{zBPD}}=0.587,95 \% \mathrm{CI}=0.459-0.751$, respectively $)$, giving the head a dolichocephalic shape. Interestingly, the BPD of the TDS controls was the smallest of all groups, leading to a smaller $\mathrm{HC}$, which was also significantly smaller than the $\mathrm{HC}$ for the TDP group $\left(\mathrm{aOR}_{\mathrm{zHC}}=0.598\right.$, 95\% CI=0.426-0.838). The heads of ASD and TDS fetuses were also proportionally smaller vs other body parts compared to TDP fetuses (Table 3), indicating that these differences are specific to the head and are not due to differences in fetal growth or fetal age. By the third trimester, these differences in biometric measures between groups had attenuated and had become statistically indistinguishable, although the BPD of the ASD fetuses had become the smallest of all groups (Table 3).

To verify that our results were not biased by the different samples in the two trimesters (Figure 1), we repeated these analyses in a subsample containing only subjects with US biometric data from both trimesters. The repeat analysis confirmed the above findings, with some differences in head size between ASD and TDP becoming even clearer in this subsample (Table 4).

Head biometric measures were consistently smaller for females than males within and across groups (Figures

2-4; Table 5), indicating a significant sex effect in fetal growth measures. Therefore, we reevaluated the group differences in biometric measures separately for males and females (Table 6). In the second trimester, the BPD in both ASD and TDS males was significantly smaller compared to TDP, but no such differences were seen in HC measures. As a result, ASD and TDS males had narrower and more elongated heads compared to TDP males $(\mathrm{zBPD}-\mathrm{zHC}=-0.271 \pm 1.04 ; \mathrm{p}=0.027$ and $\mathrm{zBPD}-\mathrm{zHC}=-0.539 \pm 1.08 ; \mathrm{p}<0.001$ for ASD-TDP and TDS-TDP, respectively). In the third trimester, the differences in head size and shape were attenuated and became statistically insignificant. In females, HC and BPD were proportionally smaller in ASD and TDS compared to

24 TDP in both pregnancy trimesters (Table 6), thus suggesting that such head shape abnormality (narrower and elongated) during mid gestation is an exclusive characteristic of males in ASD families. 
medRxiv preprint doi: https://doi.org/10.1101/2020.08.09.20170811; this version posted August 23, 2020. The copyright holder for this preprint (which was not certified by peer review) is the author/funder, who has granted medRxiv a license to display the preprint in perpetuity.

All rights reserved. No reuse allowed without permission.

\begin{tabular}{|c|c|c|c|c|c|c|}
\hline Variable & $\begin{array}{l}\text { Group } \\
\text { comparison }\end{array}$ & $\begin{array}{l}\text { Mean difference } \pm \\
\text { SD }\end{array}$ & P-value $^{a}$ & $\begin{array}{l}\text { Adjusted odds } \\
\text { ratio }(\mathrm{aOR})^{\mathrm{b}}\end{array}$ & $95 \% \mathrm{CI}$ & P-value $^{\mathbf{a}}$ \\
\hline \multicolumn{7}{|c|}{ Second Trimester: 131 ASD-TDS pairs, 116 ASD-TDP pairs } \\
\hline zBPD & $\begin{array}{l}\text { ASD }(\underline{\text { Ref }})-\text { TDS } \\
\text { ASD }(\underline{\text { Ref }})-\text { TDP } \\
\text { TDS }(\underline{\text { Ref }})-\text { TDP }\end{array}$ & $\begin{array}{l}0.420 \pm 1.63 \\
-0.402 \pm 1.53 \\
-0.870 \pm 1.74\end{array}$ & $\begin{array}{l}0.012 \\
0.018 \\
<0.001\end{array}$ & $\begin{array}{l}1.261 \\
0.685 \\
0.587\end{array}$ & $\begin{array}{l}0.998-1.594 \\
0.527-0.890 \\
0.459-0.751\end{array}$ & $\begin{array}{l}0.156 \\
\mathbf{0 . 0 1 5} \\
<\mathbf{0 . 0 0 1}\end{array}$ \\
\hline $\mathrm{zHC}$ & $\begin{array}{l}\text { ASD }(\underline{\text { Ref }})-\text { TDS } \\
\text { ASD }(\underline{\text { Ref }})-\text { TDP } \\
\text { TDS }(\underline{\text { Ref }})-\text { TDP }\end{array}$ & $\begin{array}{l}0.181 \pm 1.24 \\
-0.216 \pm 1.11 \\
0.441 \pm 1.26\end{array}$ & $\begin{array}{l}0.300 \\
0.12 \\
<\mathbf{0 . 0 0 1}\end{array}$ & $\begin{array}{l}1.087 \\
0.693 \\
0.598\end{array}$ & $\begin{array}{l}0.795-1.487 \\
0.486-0.987 \\
0.426-0.838\end{array}$ & $\begin{array}{l}1 \\
0.126 \\
\mathbf{0 . 0 0 9}\end{array}$ \\
\hline $\mathrm{zAC}$ & $\begin{array}{l}\operatorname{ASD}(\underline{\text { Ref }})-\text { TDS } \\
\operatorname{ASD}(\underline{\text { Ref }})-\text { TDP } \\
\text { TDS }(\underline{\text { Ref }})-\text { TDP }\end{array}$ & $\begin{array}{l}0.094 \pm 1.09 \\
-0.180 \pm 1.27 \\
-0.285 \pm 1.25\end{array}$ & $\begin{array}{l}0.984 \\
0.402 \\
\mathbf{0 . 0 3 6}\end{array}$ & $\begin{array}{l}0.991 \\
0.809 \\
0.792\end{array}$ & $\begin{array}{l}0.701-1.400 \\
0.595-1.100 \\
0.571-1.097\end{array}$ & $\begin{array}{l}1 \\
0.531 \\
0.480\end{array}$ \\
\hline $\mathrm{zFL}$ & $\begin{array}{l}\operatorname{ASD}(\underline{\text { Ref }})-\text { TDS } \\
\operatorname{ASD}(\underline{\text { Ref }})-\text { TDP } \\
\text { TDS }(\underline{\text { Ref }})-\text { TDP }\end{array}$ & $\begin{array}{l}-0.071 \pm 1.07 \\
-0.099 \pm 1.04 \\
-0.026 \pm 1.02\end{array}$ & $\begin{array}{l}1 \\
0.933 \\
1\end{array}$ & $\begin{array}{l}0.838 \\
0.846 \\
1.061\end{array}$ & $\begin{array}{l}0.596-1.177 \\
0.591-1.212 \\
0.733-1.536\end{array}$ & $\begin{array}{l}0.921 \\
1 \\
1\end{array}$ \\
\hline zBPD-zHC & $\begin{array}{l}\operatorname{ASD}(\underline{\text { Ref }})-\text { TDS } \\
\operatorname{ASD}(\underline{\text { Ref }})-\mathrm{TDP} \\
\mathrm{TDS}(\underline{\text { Ref }})-\mathrm{TDP}\end{array}$ & $\begin{array}{l}0.257 \pm 1.13 \\
-0.181 \pm 0.96 \\
-0.442 \pm 1.12\end{array}$ & $\begin{array}{l}\mathbf{0 . 0 3 3} \\
0.141 \\
<\mathbf{0 . 0 0 1}\end{array}$ & $\begin{array}{l}1.577 \\
0.621 \\
0.521\end{array}$ & $\begin{array}{l}1.081-2.302 \\
0.407-0.948 \\
0.364-0.746\end{array}$ & $\begin{array}{l}0.054 \\
0.081 \\
<\mathbf{0 . 0 0 1}\end{array}$ \\
\hline $\mathrm{zHC}-\mathrm{zAC}$ & $\begin{array}{l}\operatorname{ASD}(\underline{\text { Ref }})-\text { TDS } \\
\operatorname{ASD}(\underline{\text { Ref }})-\mathrm{TDP} \\
\mathrm{TDS}(\underline{\text { Ref }})-\mathrm{TDP}\end{array}$ & $\begin{array}{l}0.101 \pm 1.06 \\
-0.025 \pm 1.16 \\
-0.153 \pm 1.10\end{array}$ & $\begin{array}{l}0.837 \\
1 \\
0.369\end{array}$ & $\begin{array}{l}1.159 \\
0.939 \\
0.715\end{array}$ & $\begin{array}{l}0.811-1.657 \\
0.679-1.298 \\
0.498-1.027\end{array}$ & $\begin{array}{l}1 \\
1 \\
0.210\end{array}$ \\
\hline zBPD-zAC & $\begin{array}{l}\operatorname{ASD}(\underline{\text { Ref }})-\text { TDS } \\
\operatorname{ASD}(\underline{\text { Ref }})-\text { TDP } \\
\text { TDS }(\underline{\text { Ref }})-\text { TDP }\end{array}$ & $\begin{array}{l}0.324 \pm 1.41 \\
-0.238 \pm 1.49 \\
-0.592 \pm 1.46\end{array}$ & $\begin{array}{l}\mathbf{0 . 0 2 7} \\
0.276 \\
<\mathbf{0 . 0 0 1}\end{array}$ & $\begin{array}{l}1.369 \\
0.767 \\
0.572\end{array}$ & $\begin{array}{l}1.041-1.800 \\
0.588-1.000 \\
0.431-0.758\end{array}$ & $\begin{array}{l}0.072 \\
0.150 \\
<\mathbf{0 . 0 0 1}\end{array}$ \\
\hline $\mathrm{zHC}-\mathrm{zFL}$ & $\begin{array}{l}\operatorname{ASD}(\underline{\text { Ref }})-\text { TDS } \\
\operatorname{ASD}(\underline{\text { Ref }})-\text { TDP } \\
\text { TDS }(\underline{\text { Ref }})-\text { TDP }\end{array}$ & $\begin{array}{l}0.278 \pm 1.03 \\
-0.109 \pm 1.10 \\
-0.411 \pm 1.07\end{array}$ & $\begin{array}{l}\mathbf{0 . 0 0 9} \\
0.882 \\
<\mathbf{0 . 0 0 1}\end{array}$ & $\begin{array}{l}1.489 \\
0.813 \\
0.502\end{array}$ & $\begin{array}{l}1.009-2.197 \\
0.575-1.149 \\
0.341-0.738\end{array}$ & $\begin{array}{l}0.135 \\
0.720 \\
<\mathbf{0 . 0 0 1}\end{array}$ \\
\hline zBPD-zFL & $\begin{array}{l}\operatorname{ASD}(\underline{\text { Ref }})-\text { TDS } \\
\text { ASD }(\underline{\text { Ref }})-\text { TDP } \\
\text { TDS }(\underline{\text { Ref }})-\text { TDP }\end{array}$ & $\begin{array}{l}0.520 \pm 1.50 \\
-0.295 \pm 1.50 \\
-0.858 \pm 1.50 \\
\end{array}$ & $\begin{array}{l}<\mathbf{0 . 0 0 1} \\
0.111 \\
<\mathbf{0 . 0 0 1} \\
\end{array}$ & $\begin{array}{l}1.482 \\
0.733 \\
0.489 \\
\end{array}$ & $\begin{array}{l}1.136-1.934 \\
0.562-0.955 \\
0.368-0.650 \\
\end{array}$ & $\begin{array}{l}\mathbf{0 . 0 1 2} \\
0.063 \\
<\mathbf{0 . 0 0 1} \\
\end{array}$ \\
\hline \multicolumn{7}{|c|}{ Third Trimester: 116 ASD-TDS pairs, 116 ASD-TDP pairs } \\
\hline $\mathrm{zBPD}$ & $\begin{array}{l}\text { ASD }(\underline{\text { Ref }})-\text { TDS } \\
\text { ASD }(\underline{\text { Ref }})-\text { TDP } \\
\text { TDS }(\underline{\text { Ref }})-\text { TDP }\end{array}$ & $\begin{array}{l}0.061 \pm 2.05 \\
-0.174 \pm 2.13 \\
-0.351 \pm 2.21\end{array}$ & $\begin{array}{l}1 \\
1 \\
0.270\end{array}$ & $\begin{array}{l}0.920 \\
0.925 \\
0.985\end{array}$ & $\begin{array}{l}0.743-1.140 \\
0.773-1.106 \\
0.819-1.184\end{array}$ & $\begin{array}{l}1 \\
1 \\
1\end{array}$ \\
\hline $\mathrm{zHC}$ & $\begin{array}{l}\operatorname{ASD}(\underline{\text { Ref }})-\text { TDS } \\
\operatorname{ASD}(\underline{\text { Ref }})-\text { TDP } \\
\text { TDS }(\underline{\text { Ref }})-\text { TDP }\end{array}$ & $\begin{array}{l}0.040 \pm 1.34 \\
-0.028 \pm 1.34 \\
-0.163 \pm 1.51\end{array}$ & $\begin{array}{l}1 \\
1 \\
0.738\end{array}$ & $\begin{array}{l}0.924 \\
0.968 \\
0.977\end{array}$ & $\begin{array}{l}0.670-1.274 \\
0.729-1.287 \\
0.749-1.275\end{array}$ & $\begin{array}{l}1 \\
1 \\
1\end{array}$ \\
\hline zAC & $\begin{array}{l}\operatorname{ASD}(\underline{\text { Ref }})-\text { TDS } \\
\operatorname{ASD}(\underline{\text { Ref }})-\text { TDP } \\
\text { TDS }(\underline{\text { Ref }})-\text { TDP }\end{array}$ & $\begin{array}{l}-0.084 \pm 1.38 \\
-0.048 \pm 1.73 \\
0.019 \pm 1.73\end{array}$ & $\begin{array}{l}1 \\
1 \\
1\end{array}$ & $\begin{array}{l}0.868 \\
0.966 \\
1.050\end{array}$ & $\begin{array}{l}0.637-1.182 \\
0.777-1.202 \\
0.835-1.321\end{array}$ & $\begin{array}{l}1 \\
1 \\
1\end{array}$ \\
\hline $\mathrm{zFL}$ & $\begin{array}{l}\operatorname{ASD}(\underline{\text { Ref }})-\text { TDS } \\
\operatorname{ASD}(\underline{\text { Ref }})-\text { TDP } \\
\text { TDS }(\underline{\text { Ref }})-\text { TDP }\end{array}$ & $\begin{array}{l}-0.176 \pm 1.06 \\
0.082 \pm 1.09 \\
0.218 \pm 1.16\end{array}$ & $\begin{array}{l}0.237 \\
1 \\
0.135\end{array}$ & $\begin{array}{l}0.745 \\
1.150 \\
1.407\end{array}$ & $\begin{array}{l}0.502-1.106 \\
0.813-1.627 \\
0.994-1.992\end{array}$ & $\begin{array}{l}0.432 \\
1 \\
0.162\end{array}$ \\
\hline zBPD-zHC & $\begin{array}{l}\operatorname{ASD}(\underline{\text { Ref }})-\text { TDS } \\
\operatorname{ASD}(\underline{\text { Ref }})-\text { TDP } \\
\operatorname{TDS}(\underline{\text { Ref }})-\text { TDP }\end{array}$ & $\begin{array}{l}0.039 \pm 1.37 \\
-0.103 \pm 1.48 \\
-0.167 \pm 1.54\end{array}$ & $\begin{array}{l}1 \\
1 \\
0.735\end{array}$ & $\begin{array}{l}0.908 \\
0.910 \\
1.022\end{array}$ & $\begin{array}{l}0.651-1.266 \\
0.702-1.178 \\
0.781-1.337\end{array}$ & $\begin{array}{l}1 \\
1 \\
1\end{array}$ \\
\hline zHC-zAC & $\begin{array}{l}\operatorname{ASD}(\underline{\text { Ref }})-\text { TDS } \\
\operatorname{ASD}(\underline{\text { Ref }})-\text { TDP } \\
\text { TDS }(\underline{\text { Ref }})-\text { TDP }\end{array}$ & $\begin{array}{l}0.181 \pm 1.11 \\
0.044 \pm 1.47 \\
-0.164 \pm 1.51\end{array}$ & $\begin{array}{l}0.273 \\
1 \\
0.726\end{array}$ & $\begin{array}{l}1.244 \\
1.043 \\
0.923\end{array}$ & $\begin{array}{l}0.845-1.830 \\
0.805-1.351 \\
0.708-1.204\end{array}$ & $\begin{array}{l}0.804 \\
1 \\
1\end{array}$ \\
\hline zBPD-zAC & $\begin{array}{l}\operatorname{ASD}(\underline{\text { Ref }})-\text { TDS } \\
\operatorname{ASD}(\underline{\text { Ref }})-\text { TDP } \\
\operatorname{TDS}(\underline{\text { Ref }})-\text { TDP }\end{array}$ & $\begin{array}{l}0.094 \pm 1.70 \\
-0.097 \pm 1.89 \\
-0.307 \pm 1.95\end{array}$ & $\begin{array}{l}1 \\
1 \\
0.276\end{array}$ & $\begin{array}{l}0.922 \\
0.947 \\
0.984\end{array}$ & $\begin{array}{l}0.711-1.196 \\
0.774-1.158 \\
0.797-1.215\end{array}$ & $\begin{array}{l}1 \\
1 \\
1\end{array}$ \\
\hline $\mathrm{zHC}-\mathrm{zFL}$ & $\begin{array}{l}\operatorname{ASD}(\underline{\text { Ref }})-\text { TDS } \\
\operatorname{ASD}(\underline{\text { Ref }})-\text { TDP } \\
\text { TDS }(\underline{\text { Ref }})-\text { TDP }\end{array}$ & $\begin{array}{l}0.241 \pm 1.07 \\
-0.148 \pm 1.10 \\
-0.401 \pm 1.44\end{array}$ & $\begin{array}{l}0.063 \\
0.498 \\
\mathbf{0 . 0 0 9}\end{array}$ & $\begin{array}{l}1.235 \\
0.782 \\
0.765\end{array}$ & $\begin{array}{l}0.829-1.841 \\
0.552-1.109 \\
0.574-1.018\end{array}$ & $\begin{array}{l}0.897 \\
0.504 \\
0.198\end{array}$ \\
\hline zBPD-zFL & $\begin{array}{l}\text { ASD }(\underline{\text { Ref }})-\text { TDS } \\
\text { ASD }(\underline{\text { Ref }})-\text { TDP } \\
\text { TDS }(\underline{\text { Ref }})-\text { TDP }\end{array}$ & $\begin{array}{l}0.201 \pm 1.88 \\
-0.284 \pm 1.85 \\
-0.544 \pm 2.09\end{array}$ & $\begin{array}{l}0.792 \\
0.333 \\
\mathbf{0 . 0 1 8} \\
\end{array}$ & $\begin{array}{l}0.986 \\
0.845 \\
0.894 \\
\end{array}$ & $\begin{array}{l}0.781-1.245 \\
0.684-1.043 \\
0.734-1.088\end{array}$ & $\begin{array}{l}1 \\
0.348 \\
0.786 \\
\end{array}$ \\
\hline
\end{tabular}

2 a Paired T-test: ASD-TDS, ASD-TDP; Two-sided t-test: TDS-TDP; all p-values are Bonferroni corrected for multiple comparison (N=3)

3 . ${ }^{\mathrm{b}}$ ASD-TDS: adjusted to maternal age and gender; ASD-TDP: adjusted to maternal age; TDS-TDP: adjusted to maternal age, gender and 4 ethnicity. 
medRxiv preprint doi: https://doi.org/10.1101/2020.08.09.20170811; this version posted August 23, 2020. The copyright holder for this preprint (which was not certified by peer review) is the author/funder, who has granted medRxiv a license to display the preprint in perpetuity.

All rights reserved. No reuse allowed without permission.

\begin{tabular}{|c|c|c|c|c|c|c|}
\hline Variable & $\begin{array}{l}\text { Group } \\
\text { comparison }\end{array}$ & $\begin{array}{l}\text { Mean difference } \pm \\
\text { SD }\end{array}$ & P-value ${ }^{a}$ & $\begin{array}{l}\text { Adjusted odds } \\
\text { ratio }(\mathrm{aOR})^{\mathrm{b}}\end{array}$ & $95 \% \mathrm{CI}$ & P-value $^{a}$ \\
\hline \multicolumn{7}{|c|}{ Second Trimester } \\
\hline $\mathrm{zBPD}$ & $\begin{array}{l}\text { ASD }(\underline{\text { Ref }})-\text { TDS } \\
\operatorname{ASD}(\underline{\text { Ref }})-\text { TDP } \\
\text { TDS }(\underline{\text { Ref }})-\text { TDP }\end{array}$ & $\begin{array}{l}0.506 \pm 1.64 \\
-0.551 \pm 1.8 \\
-1.057 \pm 1.64\end{array}$ & $\begin{array}{l}0.024 \\
0.030 \\
<0.001\end{array}$ & $\begin{array}{l}1.250 \\
0.636 \\
0.463\end{array}$ & $\begin{array}{l}0.912-1.712 \\
0.462-0.874 \\
0.310-0.691\end{array}$ & $\begin{array}{l}0.411 \\
\mathbf{0 . 0 1 5} \\
<\mathbf{0 . 0 0 1}\end{array}$ \\
\hline $\mathrm{zHC}$ & $\begin{array}{l}\operatorname{ASD}(\underline{\text { Ref }})-\text { TDS } \\
\operatorname{ASD}(\underline{\text { Ref }})-\text { TDP } \\
\operatorname{TDS}(\underline{\text { Ref }})-\text { TDP }\end{array}$ & $\begin{array}{l}0.279 \pm 1.25 \\
-0.371 \pm 1.24 \\
0.650 \pm 1.21\end{array}$ & $\begin{array}{l}0.153 \\
\mathbf{0 . 0 3 9} \\
<\mathbf{0 . 0 0 1}\end{array}$ & $\begin{array}{l}1.141 \\
0.530 \\
0.382\end{array}$ & $\begin{array}{l}0.756-1.722 \\
0.338-0.832 \\
0.216-0.674\end{array}$ & $\begin{array}{l}1 \\
0.018 \\
0.003\end{array}$ \\
\hline $\mathrm{zAC}$ & $\begin{array}{l}\operatorname{ASD}(\underline{\text { Ref }})-\text { TDS } \\
\operatorname{ASD}(\underline{\text { Ref }})-\text { TDP } \\
\operatorname{TDS}(\underline{\text { Ref }})-\text { TDP }\end{array}$ & $\begin{array}{l}0.177 \pm 1.11 \\
-0.319 \pm 1.37 \\
-0.496 \pm 1.31\end{array}$ & $\begin{array}{l}0.492 \\
0.159 \\
\mathbf{0 . 0 0 9}\end{array}$ & $\begin{array}{l}1.060 \\
0.664 \\
0.569\end{array}$ & $\begin{array}{l}0.669-1.680 \\
0.440-1.003 \\
0.330-0.981\end{array}$ & $\begin{array}{l}1 \\
0.156 \\
0.129\end{array}$ \\
\hline $\mathrm{zFL}$ & $\begin{array}{l}\operatorname{ASD}(\underline{\operatorname{Ref}})-\mathrm{TDS} \\
\operatorname{ASD}(\underline{\mathrm{Re}})-\mathrm{TDP} \\
\mathrm{TDS}(\underline{\mathrm{Re}})-\mathrm{TDP}\end{array}$ & $\begin{array}{l}0.082 \pm 1.06 \\
0.053 \pm 1.07 \\
-0.029 \pm 1.00\end{array}$ & $\begin{array}{l}1 \\
1 \\
1\end{array}$ & $\begin{array}{l}1.033 \\
1.140 \\
1.019\end{array}$ & $\begin{array}{l}0.653-1.636 \\
0.710-1.829 \\
0.594-1.749\end{array}$ & $\begin{array}{l}1 \\
1 \\
1\end{array}$ \\
\hline zBPD-zHC & $\begin{array}{l}\operatorname{ASD}(\underline{\operatorname{Ref}})-\mathrm{TDS} \\
\operatorname{ASD}(\underline{\mathrm{Re}})-\mathrm{TDP} \\
\mathrm{TDS}(\underline{\mathrm{Ref}})-\mathrm{TDP}\end{array}$ & $\begin{array}{l}0.255 \pm 1.09 \\
-0.199 \pm 1.08 \\
-0.454 \pm 1.16\end{array}$ & $\begin{array}{l}0.126 \\
0.381 \\
\mathbf{0 . 0 0 9}\end{array}$ & $\begin{array}{l}1.425 \\
0.686 \\
0.552\end{array}$ & $\begin{array}{l}0.907-2.239 \\
0.421-1.116 \\
0.340-0.895\end{array}$ & $\begin{array}{l}0.375 \\
0.387 \\
\mathbf{0 . 0 4 8}\end{array}$ \\
\hline $\mathrm{zHC}-\mathrm{zAC}$ & $\begin{array}{l}\operatorname{ASD}(\underline{\text { Ref }})-\text { TDS } \\
\operatorname{ASD}(\underline{\text { Ref }})-\text { TDP } \\
\text { TDS }(\underline{\text { Ref }})-\text { TDP }\end{array}$ & $\begin{array}{l}0.104 \pm 1.04 \\
-0.039 \pm 1.28 \\
-0.143 \pm 1.16\end{array}$ & $\begin{array}{l}1 \\
1 \\
1\end{array}$ & $\begin{array}{l}1.120 \\
0.911 \\
0.756\end{array}$ & $\begin{array}{l}0.703-1.782 \\
0.610-1.359 \\
0.470-1.214\end{array}$ & $\begin{array}{l}1 \\
1 \\
0.741\end{array}$ \\
\hline zBPD-zAC & $\begin{array}{l}\operatorname{ASD}(\underline{\text { Ref }})-\text { TDS } \\
\operatorname{ASD}(\underline{\text { Ref }})-\text { TDP } \\
\text { TDS }(\underline{\text { Ref }})-\text { TDP }\end{array}$ & $\begin{array}{l}0.354 \pm 1.43 \\
-0.244 \pm 1.63 \\
-0.598 \pm 1.53\end{array}$ & $\begin{array}{l}0.093 \\
0.630 \\
\mathbf{0 . 0 0 9}\end{array}$ & $\begin{array}{l}1.300 \\
0.797 \\
0.610\end{array}$ & $\begin{array}{l}0.920-1.836 \\
0.577-1.100 \\
0.423-0.881\end{array}$ & $\begin{array}{l}0.411 \\
0.504 \\
\mathbf{0 . 0 2 4}\end{array}$ \\
\hline zHC-zFL & $\begin{array}{l}\operatorname{ASD}(\underline{\operatorname{Ref}})-\mathrm{TDS} \\
\operatorname{ASD}(\underline{\mathrm{Re}})-\mathrm{TDP} \\
\mathrm{TDS}(\underline{\mathrm{Re}})-\mathrm{TDP}\end{array}$ & $\begin{array}{l}0.194 \pm 1.02 \\
-0.417 \pm 1.07 \\
-0.611 \pm 1.01\end{array}$ & $\begin{array}{l}0.288 \\
\mathbf{0 . 0 0 3} \\
<\mathbf{0 . 0 0 1}\end{array}$ & $\begin{array}{l}1.164 \\
0.378 \\
0.283\end{array}$ & $\begin{array}{l}0.706-1.919 \\
0.219-0.654 \\
0.145-0.552\end{array}$ & $\begin{array}{l}1 \\
\mathbf{0 . 0 0 3} \\
<0.001\end{array}$ \\
\hline zBPD-zFL & $\begin{array}{l}\operatorname{ASD}(\underline{\text { Ref }})-\text { TDS } \\
\operatorname{ASD}(\underline{\text { Ref }})-\text { TDP } \\
\text { TDS }(\underline{\text { Ref }})-\text { TDP }\end{array}$ & $\begin{array}{l}0.449 \pm 1.42 \\
-0.616 \pm 1.57 \\
-1.065 \pm 1.48 \\
\end{array}$ & $\begin{array}{l}0.018 \\
\mathbf{0 . 0 0 3} \\
<\mathbf{0 . 0 0 1} \\
\end{array}$ & $\begin{array}{l}1.344 \\
0.520 \\
0.380 \\
\end{array}$ & $\begin{array}{l}0.942-1.917 \\
0.357-0.757 \\
0.238-0.608 \\
\end{array}$ & $\begin{array}{l}0.309 \\
\mathbf{0 . 0 0 3} \\
<\mathbf{0 . 0 0 1} \\
\end{array}$ \\
\hline \multicolumn{7}{|c|}{ Third Trimester } \\
\hline $\mathrm{zBPD}$ & $\begin{array}{l}\operatorname{ASD}(\underline{\text { Ref }})-\text { TDS } \\
\operatorname{ASD}(\underline{\text { Ref }})-\text { TDP } \\
\text { TDS }(\underline{\text { Ref }})-\text { TDP }\end{array}$ & $\begin{array}{l}0.029 \pm 2.30 \\
-0.579 \pm 2.45 \\
-0.608 \pm 2.32\end{array}$ & $\begin{array}{l}1 \\
0.159 \\
0.120 \\
\end{array}$ & $\begin{array}{l}0.851 \\
0.789 \\
0.876 \\
\end{array}$ & $\begin{array}{c}0.671-1.080 \\
0.631-0.988 \\
0.681-1.127\end{array}$ & $\begin{array}{l}0.552 \\
0.117 \\
0.912 \\
\end{array}$ \\
\hline $\mathrm{zHC}$ & $\begin{array}{l}\operatorname{ASD}(\underline{\text { Ref }})-\text { TDS } \\
\operatorname{ASD}(\underline{\text { Ref }})-T D P \\
\text { TDS }(\underline{\text { Ref }})-\text { TDP }\end{array}$ & $\begin{array}{l}0.144 \pm 1.42 \\
-0.269 \pm 1.34 \\
-0.414 \pm 1.49\end{array}$ & $\begin{array}{l}1 \\
0.294 \\
0.093\end{array}$ & $\begin{array}{l}0.893 \\
0.725 \\
0.755\end{array}$ & $\begin{array}{l}0.613-1.301 \\
0.483-1.088 \\
0.514-1.108\end{array}$ & $\begin{array}{l}1 \\
0.363 \\
0.450\end{array}$ \\
\hline $\mathrm{zAC}$ & $\begin{array}{l}\operatorname{ASD}(\underline{\operatorname{Ref}})-\mathrm{TDS} \\
\operatorname{ASD}(\underline{\mathrm{Re}})-\mathrm{TDP} \\
\mathrm{TDS}(\underline{\mathrm{Ref}})-\mathrm{TDP}\end{array}$ & $\begin{array}{l}0.056 \pm 1.46 \\
-0.008 \pm 1.53 \\
-0.064 \pm 1.68\end{array}$ & $\begin{array}{l}1 \\
1 \\
1\end{array}$ & $\begin{array}{l}0.915 \\
0.965 \\
0.990\end{array}$ & $\begin{array}{l}0.647-1.294 \\
0.686-1.356 \\
0.709-1.383\end{array}$ & $\begin{array}{l}1 \\
1 \\
1\end{array}$ \\
\hline $\mathrm{zFL}$ & $\begin{array}{l}\operatorname{ASD}(\underline{\text { Ref }})-\text { TDS } \\
\operatorname{ASD}(\underline{\text { Ref }})-T D P \\
\text { TDS }(\underline{\text { Ref }})-\text { TDP }\end{array}$ & $\begin{array}{l}-0.056 \pm 1.04 \\
-0.007 \pm 1.05 \\
0.049 \pm 1.13\end{array}$ & $\begin{array}{l}1 \\
1 \\
1\end{array}$ & $\begin{array}{l}0.781 \\
0.958 \\
1.121\end{array}$ & $\begin{array}{l}0.485-1.259 \\
0.591-1.551 \\
0.695-1.808\end{array}$ & $\begin{array}{l}0.933 \\
1 \\
1\end{array}$ \\
\hline zBPD-zHC & $\begin{array}{l}\operatorname{ASD}(\underline{\operatorname{Ref}})-\mathrm{TDS} \\
\operatorname{ASD}(\underline{\mathrm{Ref}})-\mathrm{TDP} \\
\mathrm{TDS}(\underline{\mathrm{Ref}})-\mathrm{TDP}\end{array}$ & $\begin{array}{l}-0.066 \pm 1.61 \\
-0.307 \pm 1.66 \\
-0.240 \pm 1.64\end{array}$ & $\begin{array}{l}1 \\
0.402 \\
0.753\end{array}$ & $\begin{array}{l}0.839 \\
0.746 \\
0.968\end{array}$ & $\begin{array}{l}0.605-1.162 \\
0.537-1.038 \\
0.680-1.376\end{array}$ & $\begin{array}{l}0.873 \\
0.249 \\
1\end{array}$ \\
\hline $\mathrm{zHC}-\mathrm{zAC}$ & $\begin{array}{l}\operatorname{ASD}(\underline{\text { Ref }})-\text { TDS } \\
\operatorname{ASD}(\underline{\text { Ref }})-T D P \\
\text { TDS }(\underline{\text { Ref }})-\text { TDP }\end{array}$ & $\begin{array}{l}0.081 \pm 1.26 \\
-0.266 \pm 1.25 \\
-0.347 \pm 1.45\end{array}$ & $\begin{array}{l}1 \\
0.246 \\
0.183\end{array}$ & $\begin{array}{l}0.994 \\
0.732 \\
0.735\end{array}$ & $\begin{array}{l}0.669-1.477 \\
0.479-1.118 \\
0.497-1.086\end{array}$ & $\begin{array}{l}1 \\
0.447 \\
0.366\end{array}$ \\
\hline zBPD-zAC & $\begin{array}{l}\operatorname{ASD}(\underline{\operatorname{Ref}})-\mathrm{TDS} \\
\operatorname{ASD}(\underline{\mathrm{Re}})-\mathrm{TDP} \\
\mathrm{TDS}(\underline{\mathrm{Re}})-\mathrm{TDP}\end{array}$ & $\begin{array}{l}-0.069 \pm 1.88 \\
-0.579 \pm 1.95 \\
-0.510 \pm 1.79\end{array}$ & $\begin{array}{l}1 \\
\mathbf{0 . 0 4 8} \\
0.078\end{array}$ & $\begin{array}{l}0.824 \\
0.709 \\
0.832\end{array}$ & $\begin{array}{l}0.624-1.089 \\
0.534-0.943 \\
0.600-1.155\end{array}$ & $\begin{array}{l}0.522 \\
0.054 \\
0.816\end{array}$ \\
\hline zHC-zFL & $\begin{array}{l}\operatorname{ASD}(\underline{\text { Ref }})-\text { TDS } \\
\operatorname{ASD}(\underline{\text { Ref }})-T D P \\
\text { TDS }(\underline{\text { Ref }})-\text { TDP }\end{array}$ & $\begin{array}{l}0.236 \pm 1.33 \\
-0.272 \pm 1.20 \\
-0.509 \pm 1.40\end{array}$ & $\begin{array}{l}0.378 \\
0.192 \\
\mathbf{0 . 0 1 5}\end{array}$ & $\begin{array}{l}1.102 \\
0.691 \\
0.640\end{array}$ & $\begin{array}{l}0.748-1.624 \\
0.440-1.087 \\
0.421-0.972\end{array}$ & $\begin{array}{l}1 \\
0.330 \\
0.108\end{array}$ \\
\hline zBPD-zFL & $\begin{array}{l}\operatorname{ASD}(\underline{\operatorname{Ref}})-\mathrm{TDS} \\
\operatorname{ASD}(\underline{\operatorname{Ref}})-\mathrm{TDP} \\
\mathrm{TDS}(\underline{\operatorname{Ref}})-\mathrm{TDP}\end{array}$ & $\begin{array}{l}0.081 \pm 2.18 \\
-0.585 \pm 2.20 \\
-0.666 \pm 2.10\end{array}$ & $\begin{array}{l}1 \\
0.090 \\
\mathbf{0 . 0 3 9}\end{array}$ & $\begin{array}{l}0.898 \\
0.751 \\
0.820\end{array}$ & $\begin{array}{l}0.707-1.142 \\
0.584-0.966 \\
0.623-1.079\end{array}$ & $\begin{array}{l}1 \\
0.078 \\
0.471\end{array}$ \\
\hline
\end{tabular}

2 a Paired T-test: ASD-TDS, ASD-TDP; Two-sided t-test: TDS-TDP; all p-values are Bonferroni corrected for multiple comparison

$3(\mathrm{~N}=3)$

4 b ASD-TDS: adjusted to maternal age and gender; ASD-TDP: adjusted to maternal age; TDS-TDP: adjusted to maternal age, gender

5 and ethnicity.

6 
medRxiv preprint doi: https://doi.org/10.1101/2020.08.09.20170811; this version posted August 23, 2020. The copyright holder for this preprint (which was not certified by peer review) is the author/funder, who has granted medRxiv a license to display the preprint in perpetuity.

All rights reserved. No reuse allowed without permission.

A.

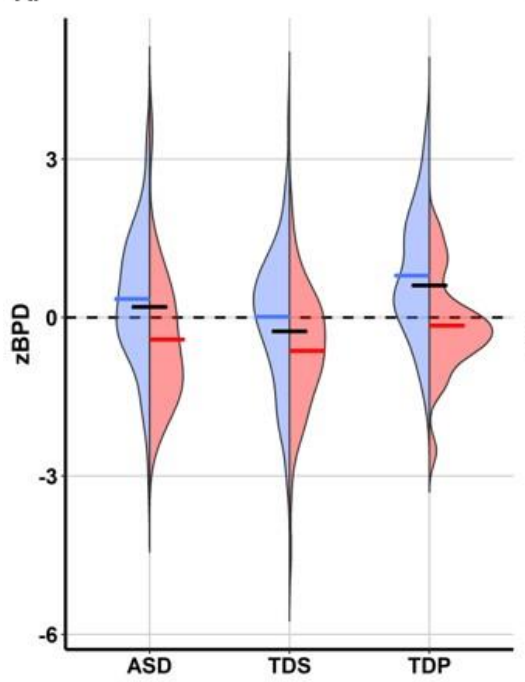

D.

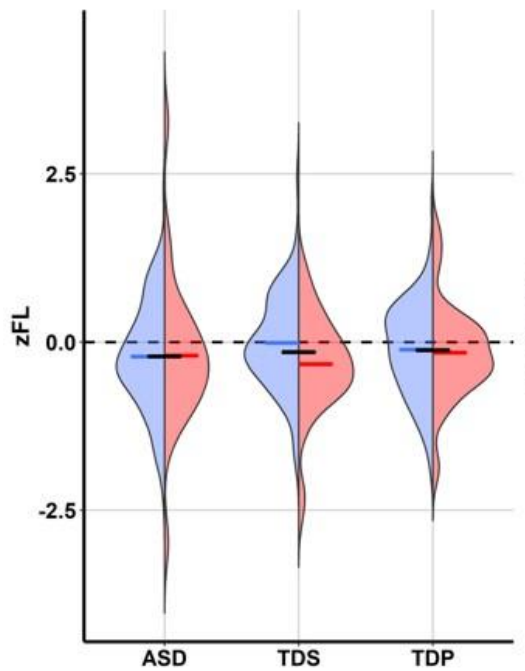

G.

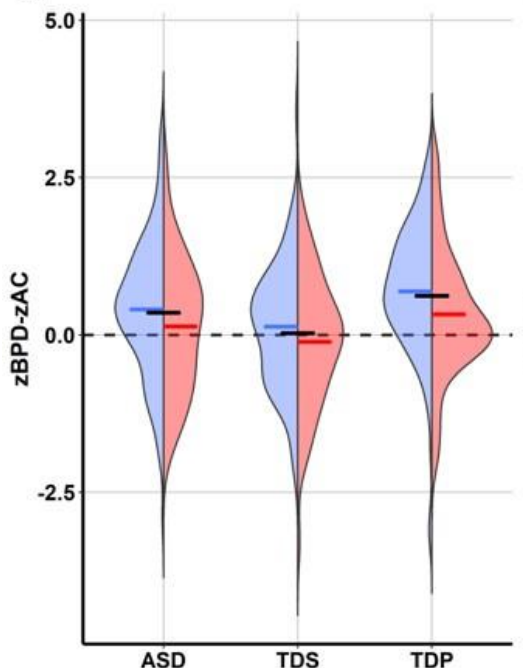

B.

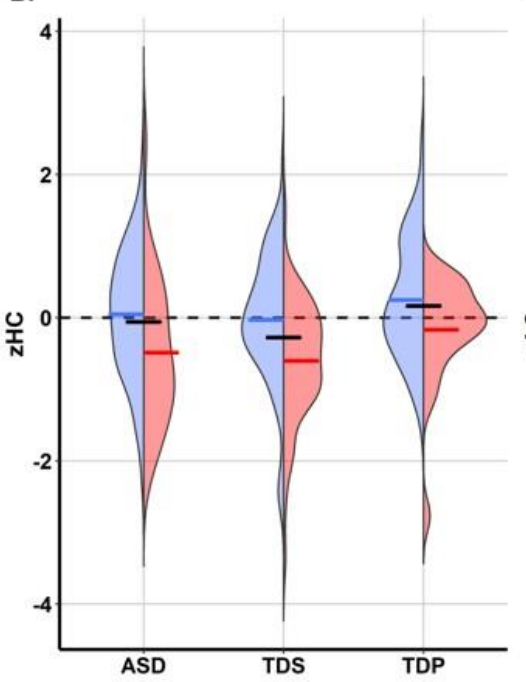

E.

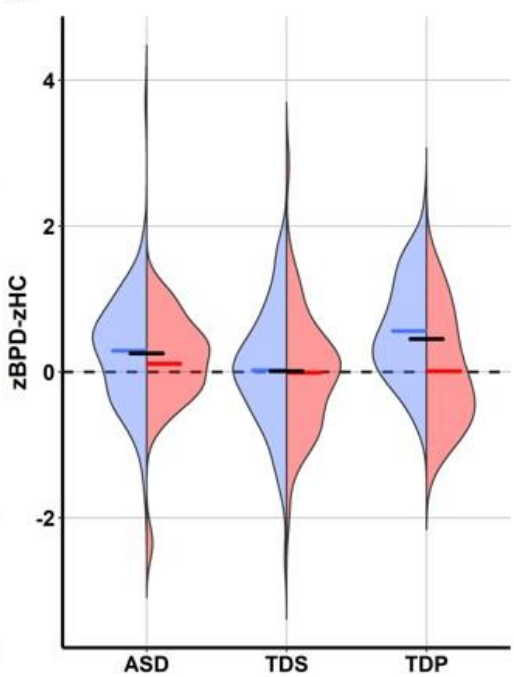

H.

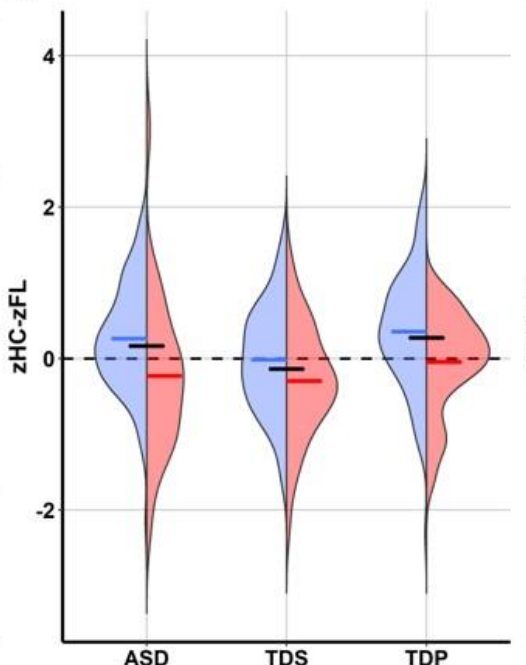

c.

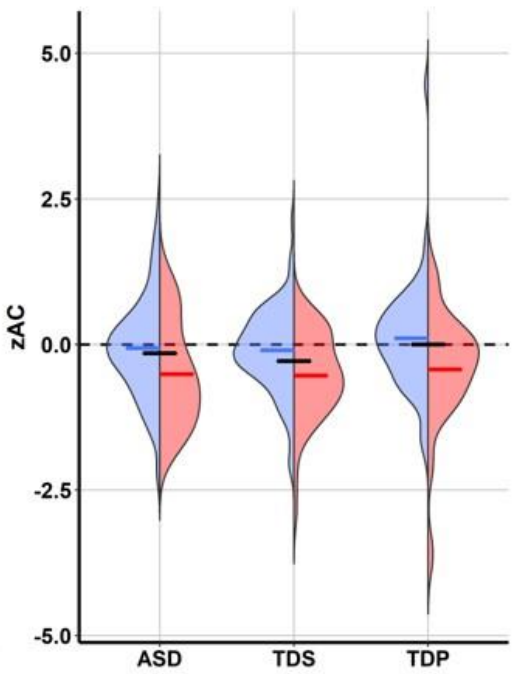

F.

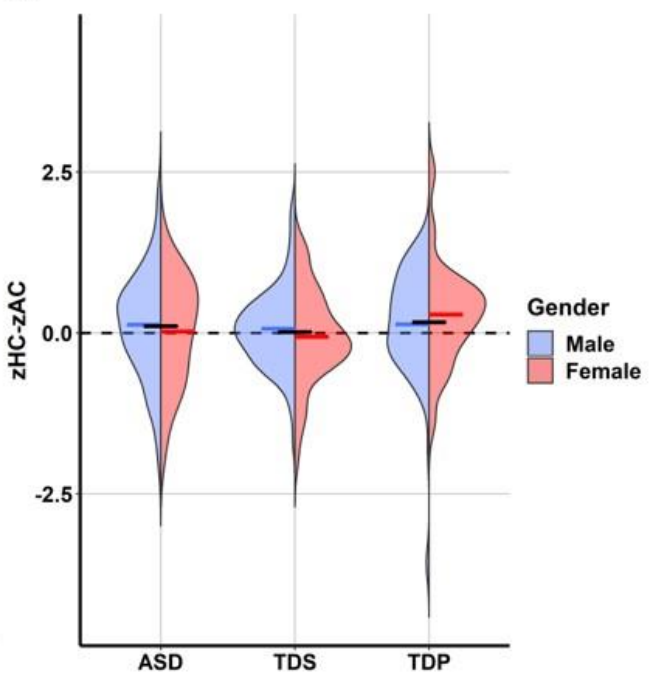

I.

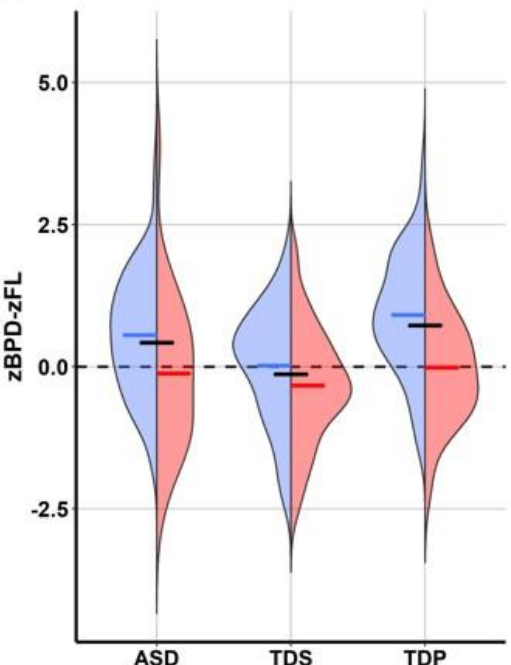

Figure 2: Half-Violin Plots of the Biometric Measures during the Second Trimester. In each panel the density (violin shape) and mean (horizontal lines) of a different biometric measure is shown for males (blue) and females (red) in the ASD, TDS and TDP groups. Black horizontal lines represent the mean measures for each group. 
medRxiv preprint doi: https://doi.org/10.1101/2020.08.09.20170811; this version posted August 23, 2020. The copyright holder for this preprint (which was not certified by peer review) is the author/funder, who has granted medRxiv a license to display the preprint in perpetuity.

All rights reserved. No reuse allowed without permission.

A.

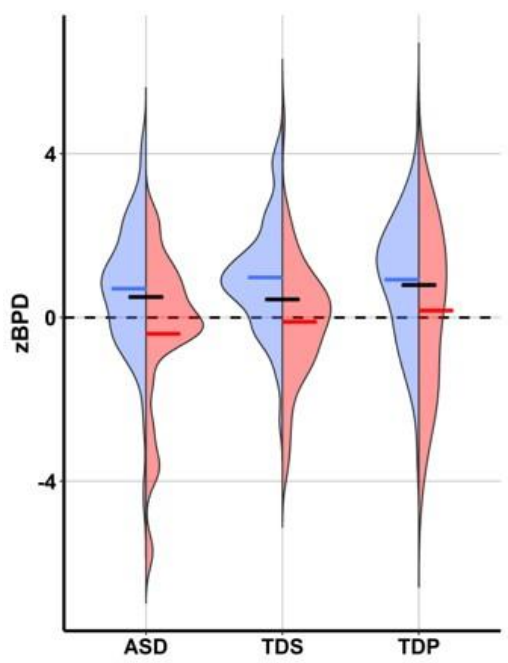

D.

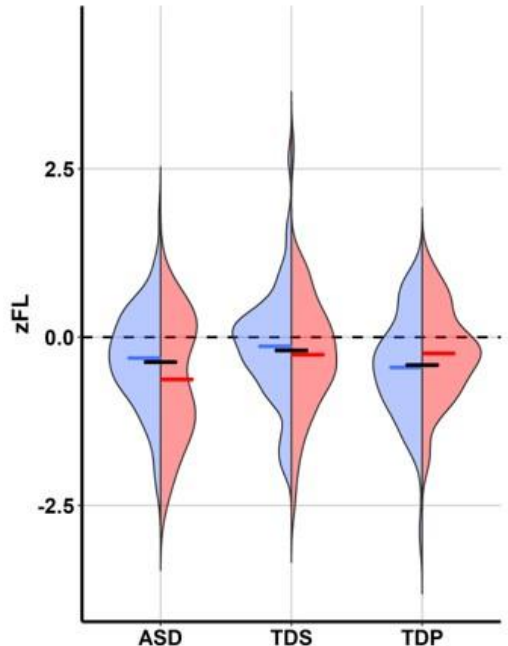

G.

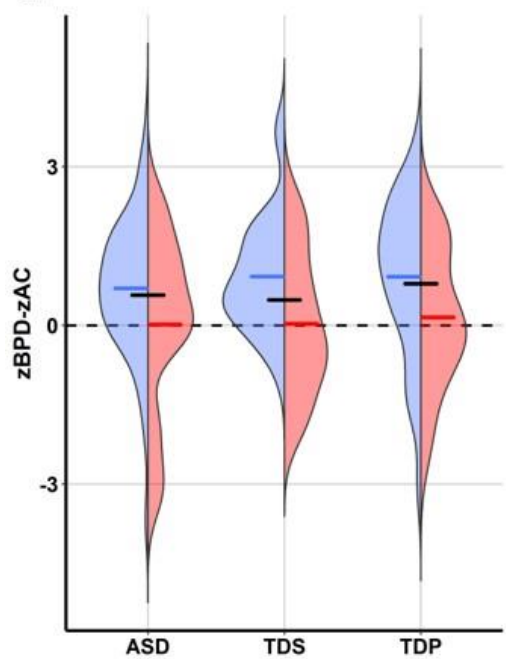

B.

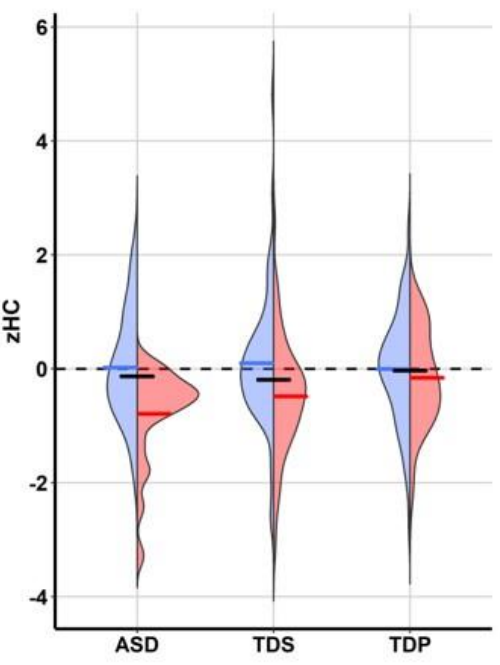

E.

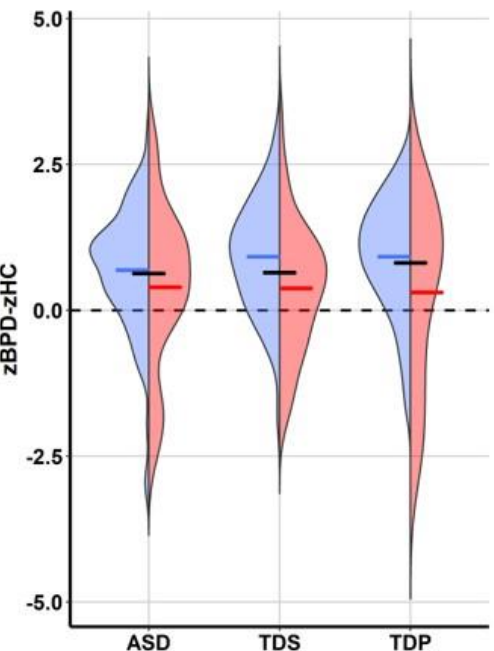

H.

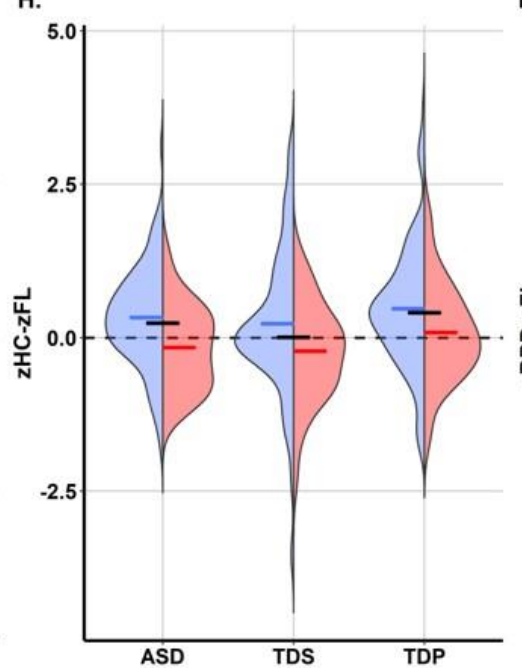

c.

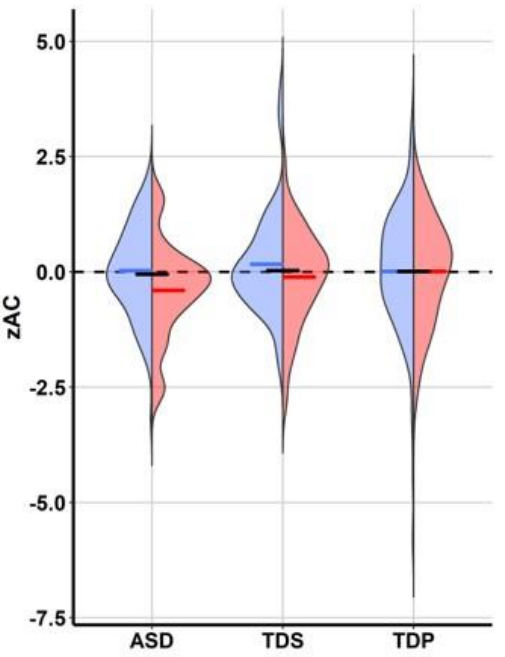

F.

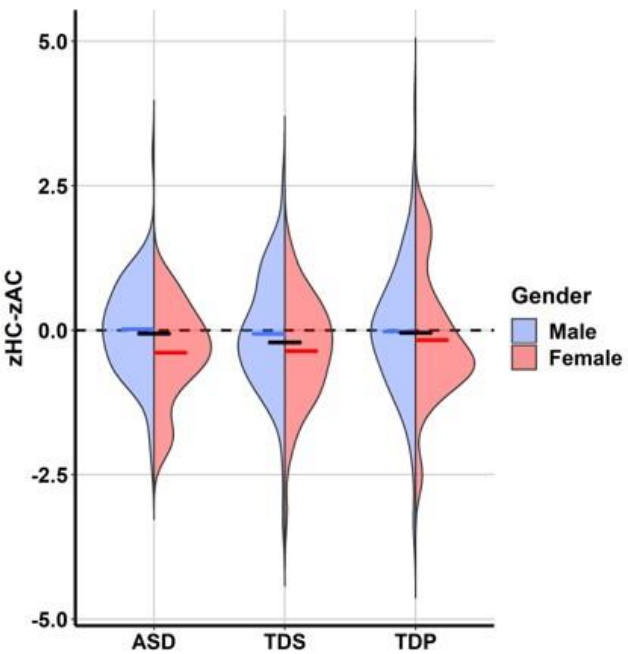

I.

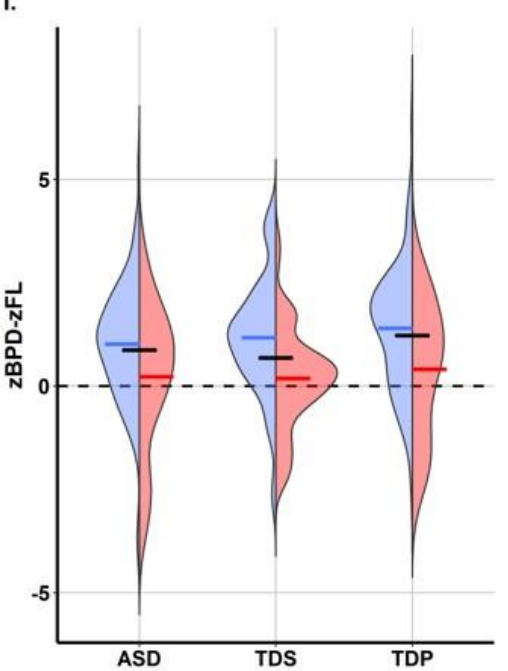

Figure 3: Half-Violin Plots of the Biometric Measures during the Third Trimester. In each panel the density (violin shape) and mean (horizontal lines) of a different biometric measure is shown for males (blue) and females (red) in the ASD, TDS and TDP groups. Black horizontal lines represent the mean measures for each group. 
medRxiv preprint doi: https://doi.org/10.1101/2020.08.09.20170811; this version posted August 23, 2020. The copyright holder for this preprint (which was not certified by peer review) is the author/funder, who has granted medRxiv a license to display the preprint in perpetuity.

All rights reserved. No reuse allowed without permission.
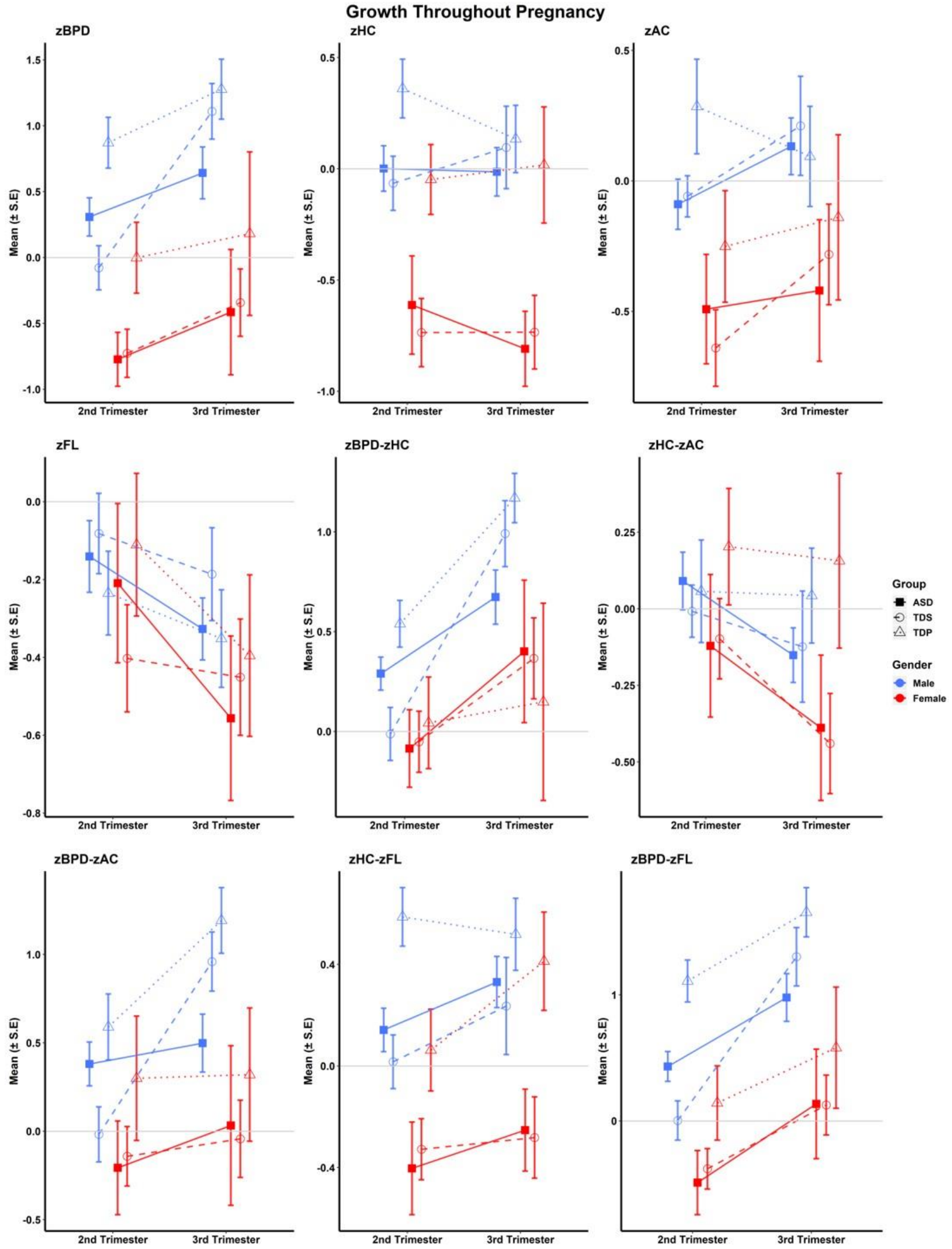

Figure 4: Changes in biometric measures between second and third trimesters. The mean and SE of different biometric measures are shown for males (blue) and females (red) in the three study groups: ASD (squares), TDS (empty circles) and TDP (empty diamonds). A steeper line between the two trimesters indicates a faster growth rate between the two trimesters. 
medRxiv preprint doi: https://doi.org/10.1101/2020.08.09.20170811; this version posted August 23, 2020. The copyright holder for this preprint (which was not certified by peer review) is the author/funder, who has granted medRxiv a license to display the preprint in

All rights reserved. No reuse allowed without permission.

\begin{tabular}{llll}
\hline \multicolumn{1}{l}{ Table 5. Sex Differences in Biometric Measures } & \multicolumn{1}{l}{$\begin{array}{l}\text { Male } \\
\text { mean (SD) }\end{array}$} & $\begin{array}{l}\text { Female } \\
\text { mean (SD) }\end{array}$ & P-value $^{\mathbf{a}}$ \\
\hline Variable & $0.408(1.26)$ & $-0.475(1.15)$ & $<\mathbf{0 . 0 0 1}$ \\
\hline Second Trimester: 277 males, 106 females & $-0.479(0.88)$ & $<\mathbf{0 . 0 0 1}$ \\
\hline zBPD & $0.093(0.88)$ & $-0.507(0.83)$ & $<\mathbf{0 . 0 0 1}$ \\
\hline zHC & $-0.018(.87)$ & $-0.262(0.84)$ & 0.127 \\
\hline zAC & $-0.127(0.75)$ & $0.026(0.77)$ & $\mathbf{0 . 0 0 2}$ \\
\hline zFL & $0.309(0.78)$ & $0.036(0.77)$ & 0.403 \\
\hline zBPD-zHC & $0.116(0.79)$ & $0.045(1.04)$ & $\mathbf{0 . 0 0 2}$ \\
\hline zHC-zAC & $0.424(1.07)$ & $-0.226(0.81)$ & $<\mathbf{0 . 0 0 1}$ \\
\hline zBPD-zAC & $0.220(0.74)$ & $-0.210(1.09)$ & $<\mathbf{0 . 0 0 1}$ \\
\hline zHC-zFL & $0.529(1.10)$ & & \\
\hline zBPD-zFL & $0.851(1.48)$ & $-0.118(1.71)$ & $<\mathbf{0 . 0 0 1}$ \\
\hline Third Trimester: 256 males, 99 females & $-0.488(0.96)$ & $<\mathbf{0 . 0 0 1}$ \\
\hline zBPD & $0.030(1.03)$ & $-0.152(1.05)$ & 0.135 \\
\hline zHC & $0.052(1.19)$ & $-0.341(0.86)$ & 0.861 \\
\hline zAC & $-0.324(0.79)$ & $0.366(1.25)$ & $\mathbf{0 . 0 0 1}$ \\
\hline zFL & $0.831(0.99)$ & $-0.329(0.91)$ & $\mathbf{0 . 0 0 8}$ \\
\hline zBPD-zHC & $-0.017(1.01)$ & $0.052(1.37)$ & $<\mathbf{0 . 0 0 1}$ \\
\hline zHC-zAC & $0.835(1.34)$ & $-0.144(0.77)$ & $<\mathbf{0 . 0 0 1}$ \\
\hline zBPD-zAC & $0.361(0.99)$ & $0.235(1.42)$ & $<\mathbf{0 . 0 0 1}$ \\
\hline zHC-zFL & $1.196(1.45)$ & & \\
\hline zBPD-zFL & & & \\
\hline & & & \\
\hline
\end{tabular}

${ }^{a}$ Two-sided t-test

\section{$\underline{\text { Longitudinal Growth }}$}

4 For approximately half the ASD fetuses and one-third of the controls there were biometric measures for both

5 the second and third trimesters (Figure 1). These data allowed us to examine differential rates of organ growth

6 during pregnancy (Figure 4; Table 7). Head width (zBPD) showed the most considerable growth compared to

7 other body parts in both cases and controls and in both males and females. However, TDS males exhibited

8 significantly faster BPD growth than both ASD and TDP males $(\beta=0.084$ and $\beta=0.100$ respectively; $p<0.001)$.

9 As a result, the gap between the TDS and TDP groups in the second trimester closed in the third trimester,

10 making the head width of male fetuses later diagnosed with ASD smaller in the third trimester vs both control

11 groups. 
medRxiv preprint doi: https://doi.org/10.1101/2020.08.09.20170811; this version posted August 23, 2020. The copyright holder for this preprint (which was not certified by peer review) is the author/funder, who has granted medRxiv a license to display the preprint in perpetuity.

All rights reserved. No reuse allowed without permission.

\begin{tabular}{|c|c|c|c|c|c|}
\hline \multicolumn{6}{|c|}{ Table 6. Group Differences in Fetal Biometric Measures for Males and Females } \\
\hline \multirow[b]{2}{*}{ Variable } & \multirow{2}{*}{$\begin{array}{l}\text { Group } \\
\text { comparison }\end{array}$} & \multicolumn{2}{|l|}{ Male } & \multicolumn{2}{|l|}{ Female } \\
\hline & & Mean difference \pm SD & P-value a,b $^{\text {P-b }}$ & Mean difference \pm SD & P-value ${ }^{a, c}$ \\
\hline \multicolumn{2}{|c|}{ Second Trimester } & \multicolumn{2}{|c|}{ N: 109 ASD, 75 TDS, 93 TDP } & \multicolumn{2}{|c|}{$\mathrm{N}: 27$ ASD, 56 TDS, 23 TDP } \\
\hline zBPD & $\begin{array}{l}\operatorname{ASD}(\underline{\operatorname{Ref}})-\mathrm{TDS} \\
\operatorname{ASD}(\underline{\operatorname{Re}})-\mathrm{TDP} \\
\mathrm{TDS}(\underline{\mathrm{Re}})-\mathrm{TDP}\end{array}$ & $\begin{array}{l}0.338 \pm 1.81 \\
-0.445 \pm 1.71 \\
-0.783 \pm 1.73\end{array}$ & $\begin{array}{l}0.222 \\
\mathbf{0 . 0 3 0} \\
<\mathbf{0 . 0 0 1}\end{array}$ & $\begin{array}{l}0.213 \pm 1.80 \\
-0.263 \pm 1.59 \\
-0.477 \pm 1.73\end{array}$ & $\begin{array}{l}1 \\
0.717 \\
0.345\end{array}$ \\
\hline zHC & $\begin{array}{l}\operatorname{ASD}(\underline{\operatorname{Ref}})-\mathrm{TDS} \\
\operatorname{ASD}(\underline{\operatorname{Re}})-\mathrm{TDP} \\
\operatorname{TDS}(\underline{\operatorname{Ref}})-\mathrm{TDP}\end{array}$ & $\begin{array}{l}0.079 \pm 1.30 \\
-0.199 \pm 1.23 \\
-0.278 \pm 1.25\end{array}$ & $\begin{array}{l}1 \\
0.315 \\
0.129\end{array}$ & $\begin{array}{l}0.115 \pm 1.37 \\
-0.321 \pm 1.28 \\
-0.436 \pm 1.28\end{array}$ & $\begin{array}{l}1 \\
0.228 \\
\mathbf{0 . 0 2 7}\end{array}$ \\
\hline $\mathrm{zAC}$ & $\begin{array}{l}\operatorname{ASD}(\underline{\text { Ref }})-\text { TDS } \\
\operatorname{ASD}(\underline{\text { Ref }})-\text { TDP } \\
\text { TDS }(\underline{\text { Ref }})-\text { TDP }\end{array}$ & $\begin{array}{l}0.036 \pm 1.16 \\
-0.170 \pm 1.30 \\
-0.206 \pm 1.24\end{array}$ & $\begin{array}{l}1 \\
0.564 \\
0.387\end{array}$ & $\begin{array}{l}0.025 \pm 1.19 \\
-0.081 \pm 1.32 \\
-0.107 \pm 1.28\end{array}$ & $\begin{array}{l}1 \\
1 \\
1\end{array}$ \\
\hline zFL & $\begin{array}{l}\operatorname{ASD}(\underline{\operatorname{Ref}})-\mathrm{TDS} \\
\operatorname{ASD}(\underline{\operatorname{Re}})-\mathrm{TDP} \\
\mathrm{TDS}(\underline{\operatorname{Re}})-\mathrm{TDP}\end{array}$ & $\begin{array}{l}-0.203 \pm 1.10 \\
-0.103 \pm 1.07 \\
0.100 \pm 1.00\end{array}$ & $\begin{array}{l}0.237 \\
1 \\
1\end{array}$ & $\begin{array}{l}0.128 \pm 1.33 \\
-0.043 \pm 1.34 \\
-0.171 \pm 1.15\end{array}$ & $\begin{array}{l}1 \\
1 \\
1\end{array}$ \\
\hline zBPD-zHC & $\begin{array}{l}\operatorname{ASD}(\underline{\text { Ref }})-\text { TDS } \\
\operatorname{ASD}(\underline{\text { Ref }})-\text { TDP } \\
\text { TDS }(\underline{\text { Ref }})-\text { TDP }\end{array}$ & $\begin{array}{l}0.268 \pm 1.14 \\
-0.271 \pm 1.04 \\
-0.539 \pm 1.08\end{array}$ & $\begin{array}{l}0.078 \\
\mathbf{0 . 0 2 7} \\
<\mathbf{0 . 0 0 1}\end{array}$ & $\begin{array}{l}0.121 \pm 1.19 \\
0.101 \pm 1.02 \\
-0.020 \pm 1.25\end{array}$ & $\begin{array}{l}0.657 \\
0.906 \\
1\end{array}$ \\
\hline zHC-zAC & $\begin{array}{l}\text { ASD }(\underline{\text { Ref }})-\text { TDS } \\
\text { ASD }(\underline{\text { Ref }})-\text { TDP } \\
\text { TDS }(\underline{\text { Ref }})-\text { TDP }\end{array}$ & $\begin{array}{l}0.060 \pm 1.09 \\
-0.008 \pm 1.22 \\
-0.068 \pm 1.12\end{array}$ & $\begin{array}{l}1 \\
1 \\
1\end{array}$ & $\begin{array}{l}0.083 \pm 1.15 \\
-0.263 \pm 1.15 \\
-0.346 \pm 1.17\end{array}$ & $\begin{array}{l}1 \\
1 \\
0.210\end{array}$ \\
\hline zBPD-zAC & $\begin{array}{l}\operatorname{ASD}(\underline{\operatorname{Ref}})-\mathrm{TDS} \\
\operatorname{ASD}(\underline{\operatorname{Re}})-\mathrm{TDP} \\
\mathrm{TDS}(\underline{\operatorname{Ref}})-\mathrm{TDP}\end{array}$ & $\begin{array}{l}0.276 \pm 1.51 \\
-0.286 \pm 1.52 \\
-0.561 \pm 1.45\end{array}$ & $\begin{array}{l}0.243 \\
0.180 \\
\mathbf{0 . 0 0 3}\end{array}$ & $\begin{array}{l}0.246 \pm 1.57 \\
-0.192 \pm 1.46 \\
-0.438 \pm 1.62\end{array}$ & $\begin{array}{l}1 \\
1 \\
0.462\end{array}$ \\
\hline zHC-zFL & $\begin{array}{l}\text { ASD }(\underline{\operatorname{Ref}})-\text { TDS } \\
\text { ASD }(\underline{\operatorname{Ref}})-\text { TDP } \\
\text { TDS }(\underline{\operatorname{Ref}})-\text { TDP }\end{array}$ & $\begin{array}{l}0.278 \pm 1.02 \\
-0.091 \pm 1.06 \\
-0.369 \pm 1.07\end{array}$ & $\begin{array}{l}\mathbf{0 . 0 3 0} \\
1 \\
\mathbf{0 . 0 0 6}\end{array}$ & $\begin{array}{l}0.068 \pm 1.29 \\
-0.184 \pm 1.25 \\
-0.252 \pm 1.12\end{array}$ & $\begin{array}{l}1 \\
0.627 \\
0.258\end{array}$ \\
\hline zBPD-zFL & $\begin{array}{l}\operatorname{ASD}(\underline{\operatorname{Ref}})-\mathrm{TDS} \\
\operatorname{ASD}(\underline{\operatorname{Re}})-\mathrm{TDP} \\
\mathrm{TDS}(\underline{\mathrm{Re}})-\mathrm{TDP}\end{array}$ & $\begin{array}{l}0.537 \pm 1.50 \\
-0.354 \pm 1.50 \\
-0.891 \pm 1.47\end{array}$ & $\begin{array}{l}\mathbf{0 . 0 0 3} \\
0.054 \\
<\mathbf{0 . 0 0 1}\end{array}$ & $\begin{array}{l}0.214 \pm 1.70 \\
-0.103 \pm 1.21 \\
-0.318 \pm 1.54\end{array}$ & $\begin{array}{l}1 \\
1 \\
0.744\end{array}$ \\
\hline$\overline{z B P D}$ & $\begin{array}{l}\text { ASD }(\underline{\operatorname{Ref}})-\mathrm{TDS} \\
\operatorname{ASD}(\underline{\operatorname{Re}})-\mathrm{TDP} \\
\mathrm{TDS}(\underline{\operatorname{Ref}})-\mathrm{TDP}\end{array}$ & $\begin{array}{l}-0.275 \pm 2.15 \\
-0.219 \pm 2.19 \\
0.056 \pm 2.12\end{array}$ & $\begin{array}{l}0.777 \\
0.978 \\
1\end{array}$ & $\begin{array}{l}-0.296 \pm 2.61 \\
-0.566 \pm 2.79 \\
-0.269 \pm 2.15\end{array}$ & $\begin{array}{l}1 \\
0.978 \\
1\end{array}$ \\
\hline $\mathrm{zHC}$ & $\begin{array}{l}\operatorname{ASD}(\underline{\operatorname{Ref}})-\mathrm{TDS} \\
\operatorname{ASD}(\underline{\operatorname{Re}})-\mathrm{TDP} \\
\operatorname{TDS}(\underline{\operatorname{Ref}})-\mathrm{TDP}\end{array}$ & $\begin{array}{l}-0.079 \pm 1.55 \\
0.023 \pm 1.39 \\
0.102 \pm 1.59\end{array}$ & $\begin{array}{l}1 \\
1 \\
1\end{array}$ & $\begin{array}{l}-0.306 \pm 1.54 \\
-0.632 \pm 1.18 \\
-0.326 \pm 1.29\end{array}$ & $\begin{array}{l}1 \\
0.186 \\
0.489\end{array}$ \\
\hline $\mathrm{zAC}$ & $\begin{array}{l}\operatorname{ASD}(\underline{\text { Ref }})-\text { TDS } \\
\operatorname{ASD}(\underline{\text { Ref }})-\text { TDP } \\
\text { TDS }(\underline{\text { Ref }})-\text { TDP }\end{array}$ & $\begin{array}{l}-0.140 \pm 1.51 \\
0.015 \pm 1.71 \\
0.155 \pm 1.90\end{array}$ & $\begin{array}{l}1 \\
1 \\
1\end{array}$ & $\begin{array}{l}-0.288 \pm 1.65 \\
-0.412 \pm 1.50 \\
-0.124 \pm 1.39\end{array}$ & $\begin{array}{l}0.579 \\
0.393 \\
1\end{array}$ \\
\hline $\mathrm{zFL}$ & $\begin{array}{l}\text { ASD }(\underline{\text { Ref }})-\text { TDS } \\
\text { ASD }(\underline{\text { Ref }})-\text { TDP } \\
\text { TDS }(\underline{\text { Ref }})-\text { TDP }\end{array}$ & $\begin{array}{l}-0.176 \pm 1.13 \\
0.140 \pm 1.08 \\
0.316 \pm 1.17\end{array}$ & $\begin{array}{l}0.516 \\
0.603 \\
0.057\end{array}$ & $\begin{array}{l}-0.368 \pm 1.41 \\
-0.387 \pm 1.12 \\
-0.192 \pm 1.12\end{array}$ & $\begin{array}{l}0.495 \\
0.681 \\
1\end{array}$ \\
\hline zBPD-zHC & $\begin{array}{l}\text { ASD }(\underline{\text { Ref }})-\text { TDS } \\
\text { ASD }(\underline{\text { Ref }})-\text { TDP } \\
\text { TDS }(\underline{\text { Ref }})-\text { TDP }\end{array}$ & $\begin{array}{l}-0.231 \pm 1.50 \\
-0.231 \pm 1.44 \\
-0.0002 \pm 1.42\end{array}$ & $\begin{array}{l}0.522 \\
0.348 \\
1\end{array}$ & $\begin{array}{l}0.016 \pm 1.86 \\
0.086 \pm 2.07 \\
0.071 \pm 1.62\end{array}$ & $\begin{array}{l}1 \\
1 \\
1\end{array}$ \\
\hline zHC-zAC & $\begin{array}{l}\operatorname{ASD}(\underline{\operatorname{Ref}})-\mathrm{TDS} \\
\operatorname{ASD}(\underline{\operatorname{Re}})-\mathrm{TDP} \\
\mathrm{TDS}(\underline{\mathrm{Re}})-\mathrm{TDP}\end{array}$ & $\begin{array}{l}0.081 \pm 1.35 \\
0.039 \pm 1.45 \\
-0.042 \pm 1.64\end{array}$ & $\begin{array}{l}1 \\
1 \\
1\end{array}$ & $\begin{array}{l}-0.029 \pm 1.38 \\
-0.220 \pm 1.33 \\
-0.191 \pm 1.24\end{array}$ & $\begin{array}{l}1 \\
1 \\
1\end{array}$ \\
\hline zBPD-zAC & $\begin{array}{l}\operatorname{ASD}(\underline{\text { Ref }})-\text { TDS } \\
\text { ASD }(\underline{\text { Ref }})-\text { TDP } \\
\text { TDS }(\underline{\text { Ref }})-\text { TDP }\end{array}$ & $\begin{array}{l}-0.223 \pm 1.82 \\
-0.220 \pm 2.01 \\
0.003 \pm 1.98\end{array}$ & $\begin{array}{l}0.837 \\
0.843 \\
1\end{array}$ & $\begin{array}{l}-0.013 \pm 2.22 \\
-0.134 \pm 2.10 \\
-0.121 \pm 1.71\end{array}$ & $\begin{array}{l}1 \\
1 \\
1\end{array}$ \\
\hline zHC-zFL & $\begin{array}{l}\operatorname{ASD}(\underline{\operatorname{Ref}})-\mathrm{TDS} \\
\operatorname{ASD}(\underline{\operatorname{Re}})-\mathrm{TDP} \\
\mathrm{TDS}(\underline{\mathrm{Re}})-\mathrm{TDP}\end{array}$ & $\begin{array}{l}0.102 \pm 1.45 \\
-0.143 \pm 1.32 \\
-0.245 \pm 1.60\end{array}$ & $\begin{array}{l}1 \\
0.858 \\
0.540\end{array}$ & $\begin{array}{l}0.059 \pm 1.21 \\
-0.244 \pm 1.03 \\
-0.303 \pm 1.05\end{array}$ & $\begin{array}{l}1 \\
0.771 \\
0.534\end{array}$ \\
\hline zBPD-zFL & $\begin{array}{l}\operatorname{ASD}(\underline{\operatorname{Ref}})-\mathrm{TDS} \\
\operatorname{ASD}(\underline{\operatorname{Re}})-\mathrm{TDP} \\
\mathrm{TDS}(\underline{\mathrm{Re}})-\mathrm{TDP}\end{array}$ & $\begin{array}{l}-0.153 \pm 2.11 \\
-0.378 \pm 2.08 \\
-0.225 \pm 2.12\end{array}$ & $\begin{array}{l}1 \\
0.225 \\
1\end{array}$ & $\begin{array}{l}0.044 \pm 2.19 \\
-0.178 \pm 2.37 \\
-0.222 \pm 1.75\end{array}$ & $\begin{array}{l}1 \\
1 \\
0.888\end{array}$ \\
\hline
\end{tabular}

$2 \quad{ }^{\mathrm{a}} \mathrm{P}$-value after Bonferroni correction for multiple comparisons $(\mathrm{N}=3) ;{ }^{\mathrm{b}}$ Two-sided t-test; ${ }^{\mathrm{c}}$ Mann-Whitney U test. 
medRxiv preprint doi: https://doi.org/10.1101/2020.08.09.20170811; this version posted August 23, 2020. The copyright holder for this preprint (which was not certified by peer review) is the author/funder, who has granted medRxiv a license to display the preprint in perpetuity.

All rights reserved. No reuse allowed without permission.

\begin{tabular}{|c|c|c|c|c|c|c|c|}
\hline \multirow[b]{2}{*}{ Variable } & \multirow{2}{*}{$\begin{array}{l}\text { Group } \\
\text { comparison }^{a}\end{array}$} & \multicolumn{3}{|l|}{ Male } & \multicolumn{3}{|l|}{ Female } \\
\hline & & Adjusted $\beta^{b}$ & $95 \% \mathrm{CI}$ & P-value & Adjusted $\beta^{b}$ & $95 \% \mathrm{CI}$ & P-value \\
\hline zBPD & $\begin{array}{l}\operatorname{ASD}(\underline{\text { Ref }})-\text { TDS } \\
\operatorname{ASD}(\underline{\text { Ref }})-\text { TDP } \\
\text { TDP }(\underline{\text { Ref }})-\text { TDS }\end{array}$ & $\begin{array}{l}0.084 \\
<0.001 \\
0.100\end{array}$ & $\begin{array}{l}0.041-0.127 \\
-0.038-0.038 \\
0.052-0.147\end{array}$ & $\begin{array}{l}<\mathbf{0 . 0 0 1} \\
1 \\
<\mathbf{0 . 0 0 1}\end{array}$ & $\begin{array}{l}-0.010 \\
-0.056 \\
0.030\end{array}$ & $\begin{array}{l}-0.087-0.067 \\
-0.166-0.055 \\
-0.057-0.116\end{array}$ & $\begin{array}{l}1 \\
0.951 \\
1\end{array}$ \\
\hline $\mathrm{zHC}$ & $\begin{array}{l}\operatorname{ASD}(\underline{\text { Ref }})-\text { TDS } \\
\operatorname{ASD}(\underline{\text { Ref }})-\text { TDP } \\
\text { TDP }(\underline{\text { Ref }})-\text { TDS }\end{array}$ & $\begin{array}{l}0.013 \\
-0.035 \\
0.092\end{array}$ & $\begin{array}{l}-0.017-0.043 \\
-0.063--0.007 \\
0.041-0.143\end{array}$ & $\begin{array}{l}1 \\
0.045 \\
0.012\end{array}$ & $\begin{array}{l}0.022 \\
0.035 \\
-0.009\end{array}$ & $\begin{array}{l}-0.032-0.077 \\
-0.026-0.096 \\
-0.067-0.049\end{array}$ & $\begin{array}{l}1 \\
0.783 \\
1\end{array}$ \\
\hline $\mathrm{zAC}$ & $\begin{array}{l}\operatorname{ASD}(\underline{\text { Ref }})-\mathrm{TDS} \\
\operatorname{ASD}(\underline{\text { Ref }})-\mathrm{TDP} \\
\mathrm{TDP}(\underline{\operatorname{Ref}})-\mathrm{TDS}\end{array}$ & $\begin{array}{l}<-0.001 \\
-0.043 \\
0.054\end{array}$ & $\begin{array}{l}-0.028-0.028 \\
-0.071--0.014 \\
0.013-0.095\end{array}$ & $\begin{array}{l}1 \\
\mathbf{0 . 0 0 9} \\
\mathbf{0 . 0 3 0}\end{array}$ & $\begin{array}{l}0.017 \\
-0.013 \\
0.033\end{array}$ & $\begin{array}{l}-0.021-0.056 \\
-0.078-0.053 \\
-0.017-0.084\end{array}$ & $\begin{array}{l}1 \\
1 \\
0.549\end{array}$ \\
\hline $\mathrm{zFL}$ & $\begin{array}{l}\operatorname{ASD}(\underline{\operatorname{Ref}})-\mathrm{TDS} \\
\operatorname{ASD}(\underline{\operatorname{Re}})-\mathrm{TDP} \\
\mathrm{TDP}(\underline{\operatorname{Ref}})-\mathrm{TDS}\end{array}$ & $\begin{array}{l}-0.001 \\
-0.003 \\
-0.007\end{array}$ & $\begin{array}{r}-0.024-0.023 \\
-0.027-0.021 \\
-0.033-0.020\end{array}$ & $\begin{array}{l}1 \\
1 \\
0.617\end{array}$ & $\begin{array}{l}-0.008 \\
-0.005 \\
-0.001\end{array}$ & $\begin{array}{r}-0.046-0.030 \\
-0.059-0.050 \\
-0.047-0.044\end{array}$ & $\begin{array}{l}1 \\
1 \\
1\end{array}$ \\
\hline zBPD-zHC & $\begin{array}{l}\operatorname{ASD}(\underline{\operatorname{Ref}})-\mathrm{TDS} \\
\operatorname{ASD}(\underline{\operatorname{Ref}})-\mathrm{TDP} \\
\mathrm{TDP}(\underline{\operatorname{Ref}})-\mathrm{TDS}\end{array}$ & $\begin{array}{l}0.025 \\
0.024 \\
0.031\end{array}$ & $\begin{array}{l}0.006-0.044 \\
-0.005-0.053 \\
-0.093-0.155\end{array}$ & $\begin{array}{l}\mathbf{0 . 0 1 8} \\
0.318 \\
0.687\end{array}$ & $\begin{array}{l}-0.004 \\
-0.077 \\
0.084\end{array}$ & $\begin{array}{l}-0.060-0.052 \\
-0.165-0.011 \\
0.012-0.156\end{array}$ & $\begin{array}{l}1 \\
0.252 \\
0.069\end{array}$ \\
\hline $\mathrm{zHC}-\mathrm{zAC}$ & $\begin{array}{l}\operatorname{ASD}(\underline{\text { Ref }})-\text { TDS } \\
\operatorname{ASD}(\underline{\text { Ref }})-\text { TDP } \\
\text { TDP }(\underline{\text { Ref }})-\text { TDS }\end{array}$ & $\begin{array}{l}-0.002 \\
0.023 \\
-0.038\end{array}$ & $\begin{array}{r}-0.036-0.032 \\
-0.012-0.058 \\
-0.085-0.008\end{array}$ & $\begin{array}{l}1 \\
0.576 \\
0.312\end{array}$ & $\begin{array}{l}-0.052 \\
0.057 \\
-0.032\end{array}$ & $\begin{array}{l}-0.087-0.017 \\
-0.013-0.128 \\
-0.086-0.021\end{array}$ & $\begin{array}{l}\mathbf{0 . 0 1 2} \\
0.318 \\
0.699\end{array}$ \\
\hline zBPD-zAC & $\begin{array}{l}\operatorname{ASD}(\underline{\text { Ref }})-\text { TDS } \\
\operatorname{ASD}(\underline{\text { Ref }})-\text { TDP } \\
\text { TDP }(\underline{\text { Ref }})-\text { TDS }\end{array}$ & $\begin{array}{l}0.089 \\
0.001 \\
0.041\end{array}$ & $\begin{array}{l}0.046-0.132 \\
-0.037-0.039 \\
-0.011-0.094\end{array}$ & $\begin{array}{l}<0.001 \\
1 \\
0.366\end{array}$ & $\begin{array}{l}0.021 \\
-0.044 \\
0.056\end{array}$ & $\begin{array}{l}-0.050-0.092 \\
-0.147-0.060 \\
-0.018-0.130\end{array}$ & $\begin{array}{l}1 \\
1 \\
0.384\end{array}$ \\
\hline zHC-zFL & $\begin{array}{l}\operatorname{ASD}(\underline{\operatorname{Ref}})-\mathrm{TDS} \\
\operatorname{ASD}(\underline{\text { Ref }})-\mathrm{TDP} \\
\mathrm{TDP}(\underline{\operatorname{Ref}})-\mathrm{TDS}\end{array}$ & $\begin{array}{l}0.014 \\
-0.025 \\
0.039\end{array}$ & $\begin{array}{l}-0.018-0.046 \\
-0.055-0.006 \\
0.005-0.074\end{array}$ & $\begin{array}{l}1 \\
0.324 \\
0.081\end{array}$ & $\begin{array}{l}0.029 \\
0.051 \\
-0.017\end{array}$ & $\begin{array}{l}-0.002-0.060 \\
-0.0004-0.103 \\
-0.072-0.038\end{array}$ & $\begin{array}{l}0.201 \\
0.156 \\
1\end{array}$ \\
\hline zBPD-zFL & $\begin{array}{l}\operatorname{ASD}(\underline{\operatorname{Ref}})-\mathrm{TDS} \\
\operatorname{ASD}(\underline{\operatorname{Re}})-\mathrm{TDP} \\
\mathrm{TDP}(\underline{\operatorname{Ref}})-\mathrm{TDS}\end{array}$ & $\begin{array}{l}0.095 \\
0.001 \\
0.088\end{array}$ & $\begin{array}{l}0.051-0.138 \\
-0.037-0.039 \\
0.041-0.135\end{array}$ & $\begin{array}{l}<0.001 \\
1 \\
<0.001\end{array}$ & $\begin{array}{l}0.002 \\
-0.044 \\
0.031\end{array}$ & $\begin{array}{l}-0.075-0.078 \\
-0.147-0.060 \\
-0.049-0.112\end{array}$ & $\begin{array}{l}1 \\
1 \\
1\end{array}$ \\
\hline
\end{tabular}

a 85 ASD (70 males, 15 females), 69 TDS ( 36 males, 33 females), 56 TDP (43 males, 13 females).

${ }^{b}$ Interaction of ASD diagnosis with pregnancy week assessed by linear mixed effect regression adjusted to ethnicity. Positive $\beta$ means faster growth rate compared to reference group.

${ }^{c} \mathrm{P}$-value after Bonferroni correction for multiple comparisons $(\mathrm{N}=3)$.

\section{$\underline{\text { Association with ASD Phenotypes }}$}

Finally, we examined the association between fetal biometric measures and the severity of ASD symptoms (Figure 5). Three major trends were seen in this analyses: 1) smaller biometric measures (i.e. HC, BPD, AC, and FL) were associated with worse ASD symptoms especially among males in the second trimester (Figure 5A) and females in third trimester (Figure 5D); 2) larger head-to-body ratios were positively correlated with more severe ASD symptoms, especially in the second trimester in both males and females (Figures 5A and 5B respectively); and 3) In females, different trends were seen between the two trimesters. In the second trimester, most biometric measures were positively correlated with ASD severity (Figure 5C), while in the third trimester, they were inversely correlated with symptom severity (Figure 5D). Of note, none of these correlations remained statistically significant after Bonferroni correction for multiple testing. 
A.

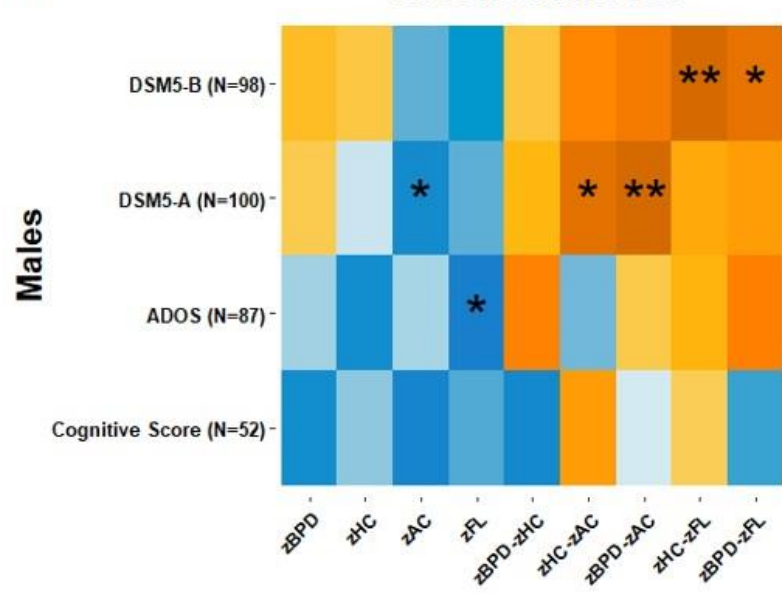

C.

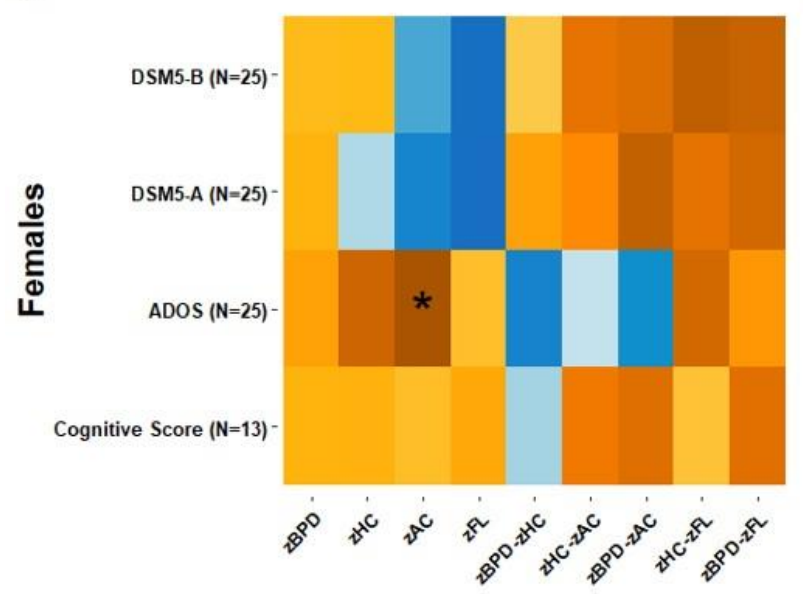

B.

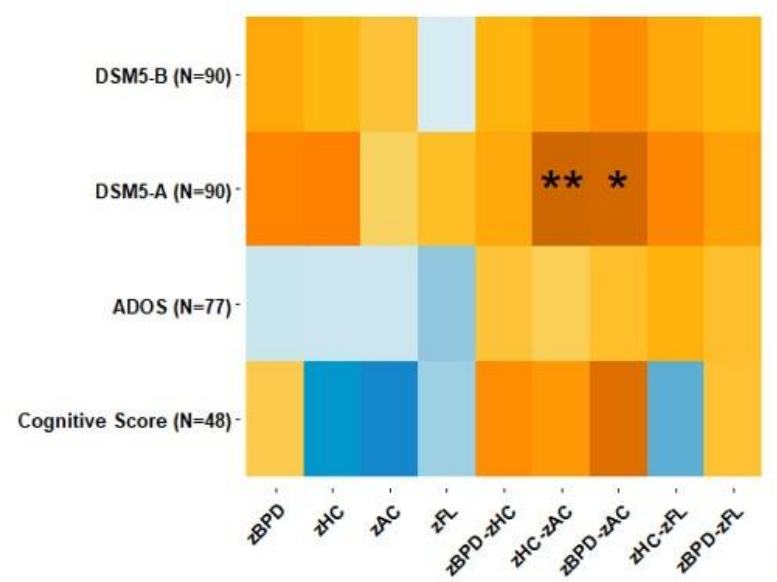

D.

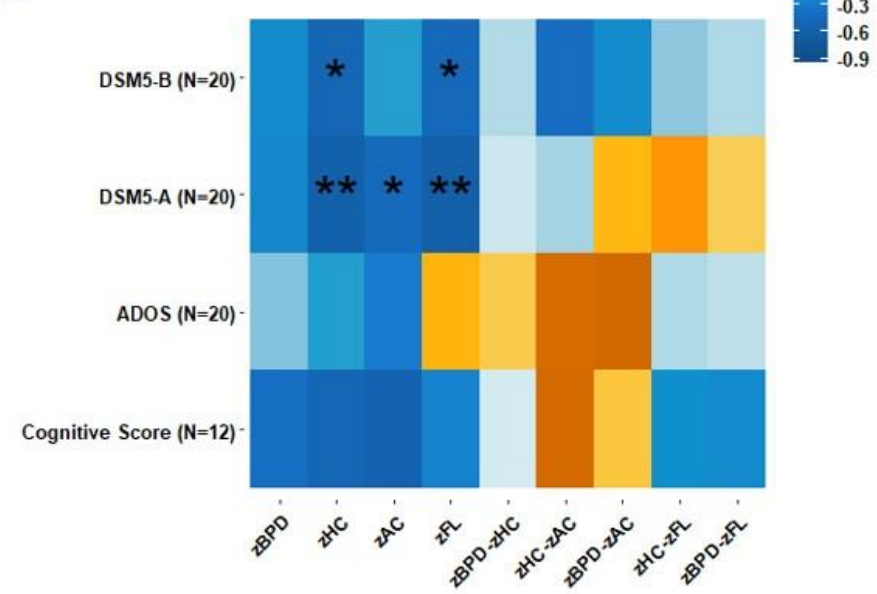

Figure 5: Correlation between ASD Severity and Fetal Biometric Measures in the Second and Third Trimesters of pregnancy in Males and Females. Spearman correlation coefficients (R) are color coded, with asterisks indicating statistically significant correlations: * P-value $<0.05$, ** P-value $\leq 0.01$. For all tests, a positive $\mathrm{R}$ means a larger biometric measure is correlated with worse outcome of specific clinical test, as follows:DSM5-B: Restrictive-Repetitive Domain has a range of three values (1) Requiring Support, (2) Requiring Substantial Support, (3) Requiring Very Substantial Support. A higher score means a worse outcome. DSM5-A: Social Domain has a range of three values (1) Requiring Support, (2) Requiring Substantial Support, (3) Requiring Very Substantial Support. A higher score means worse outcome. ADOS: A higher score means a worse outcome. Cognitive Score: Cognitive abilities of child. In the original test. a higher score means better outcome. In order to avoid confusion, we multiplied correlation outcome by -1 so that in this test too, a positive correlation means a worse outcome.

\section{Discussion}

13 This is the largest and most comprehensive study to date to examine the association between fetal growth

14 parameters and ASD. Three main findings (discussed in detail below) emerged from this study. First, fetuses later diagnosed with ASD and their TDS have narrower heads during mid-gestation compared to TDP, thus suggesting that such fetal head growth abnormality is a familial trait of ASD. Second, ASD-related head growth 
medRxiv preprint doi: https://doi.org/10.1101/2020.08.09.20170811; this version posted August 23, 2020. The copyright holder for this preprint (which was not certified by peer review) is the author/funder, who has granted medRxiv a license to display the preprint in All rights reserved. No reuse allowed without permission.

abnormalities are modulated by the sex of the fetus, with males and females showing different head shapes during gestation. Third, fetal head anomalies appear to be associated with the severity of ASD.

The abnormal fetal head growth associated with ASD that we report in this study suggests that brain abnormalities associated with ASD begin during gestation. Further support for prenatal brain anomalies in ASD can be found in a recent review which summarizes findings from multiple studies of ASD about abnormal prenatal development of the brain including cell proliferation, neurogenesis, migration, laminar organization, and neurite outgrowth during the first and second trimester alongside neurite outgrowth, synaptogenesis, and synapse functioning in the third trimester and early postnatal life. ${ }^{10}$ Additional support may be drawn from postmortem studies that found clues of aberrant neuronal migration during early prenatal brain development in the prefrontal and temporal cortical tissue, alongside evidence of curtailment of maturation of the forebrain limbic system and abnormalities in the cerebellar circuits, all of which are brain regions held to be affected ASD.$^{5,6,8,9}$ In addition, two postnatal MRI studies in preterm infants indicated structural brain asymmetry and reduced brain volume in preterm fetuses later diagnosed with ASD, again suggesting that abnormal head growth begins early in prenatal life, though ASD in the context of extreme prematurity might have a different pathogenic pathway compared to ASD in the general population..$^{54,55}$ Moreover, a recent MRI study found a $15 \%$ increase of extra-axial CSF volume among ASD children during early childhood. ${ }^{19}$ This increase is categorised as benign external hydrocephalus, characterized by increased CSF mainley above the frontal lobes, and is suggested to accure due to delayed maturation of arachnoid granulations which leads to an imbalance between the production and absorption of CSF.${ }^{56}$ Our finding of a narrow and elongated head among ASD males is consistent with this fingdings, and may suggest the increase of CSF above the frontal lobes may begin during prenatal life due to the delayed maturation of arachnoid granulations, leading to the abnormal and elongated head shape.

Our findings suggest that abnormalities in the fetal head during gestation that are later associated with ASD is a familial trait. Support for this premise can be drawn both from a prospective study showing smaller fetal heads in families with ASD compared to fetuses from families without $\mathrm{ASD}^{36}$ and from a study that found an association between smaller $\mathrm{HC}$ and various autistic traits ${ }^{37}$, which are more prevalent in ASD families. ${ }^{57}$ Additional support comes from genetic studies suggesting that $\mathrm{HC}$ is highly heritable ${ }^{58}$ and that genetic variations associated with $\mathrm{HC}$ are also associated with risk of ASD. ${ }^{59}$ Another indication of a familial link 
medRxiv preprint doi: https://doi.org/10.1101/2020.08.09.20170811; this version posted August 23, 2020. The copyright holder for this preprint (which was not certified by peer review) is the author/funder, who has granted medRxiv a license to display the preprint in All rights reserved. No reuse allowed without permission.

between abnormal fetal head growth and risk of ASD lies in some maternal endocrine functions during pregnancy that have been associated with both of these traits. ${ }^{60,61}$ Interestingly, fetuses of our TDS group demonstrated accelerated head growth during late gestation to compensate for their relatively smaller heads, a finding consistent with previous study demonstrating slightly faster growth rate among TDS compared to TDP. ${ }^{36}$

5 Similar accelerated head growth in early infancy has been reported in ASD children, ${ }^{7,11-15}$ providing another familial trait of head growth abnormality associated with ASD. Furthermore, adjustment of brain developmental rate has also been shown as type of whole-brain adaptation in response to early environmental adversity in order to maximize its fit with external enviorment. ${ }^{62}$

Our findings also suggest that ASD is associated with different prenatal head growth abnormalities in males and females. Similar sex-specific head size abnormalities were seen in a large population-based study at birth and throughout early childhood in children with ASD.${ }^{18}$ In addition, differences in total brain volume, and in gray and white matter volumes in different brain regions were seen throughout childhood in boys and girls with ASD. ${ }^{14,16,18,22,28-30}$ Furthermore, our study suggests that the observed sex-specific prenatal head growth abnormalities in fetuses later diagnosed with ASD are correlated with symptom severity in both sexes. This finding is consistent with previous postnatal studies that reported correlations between postnatal head size, brain morphometry, and extra-axial CSF volume on the one hand, to behavioral development, IQ, ${ }^{21}$ social deficits, ${ }^{15}$ sleep disturbances, lower non-verbal ability, poorer motor ability, ${ }^{19}$ and ADOS scores,${ }^{22}$ on the other. We therefore suggest that postnatal sex-specific differences in ASD severity are associated with differences in fetal brain development between males and females later diagnosed with ASD.

Our study has several advantages over previous investigations of prenatal US data of children with ASD. The use of two distinct control groups, TDS and TDP, enabled us to adjust our findings to multiple familial and prenatal confounders that are known to have a considerable effect on both ASD risk and fetal growth (e.g., $\operatorname{sex}^{26,40}$, shared genetics among siblings $\left.{ }^{1,2,38,39}\right)$, making our findings more compelling. In this context, previous findings suggesting larger $\mathrm{HC}^{34}$ or $\mathrm{BPD}^{33}$ in ASD fetuses may be attributed to bias resulting from the large and unaddressed proportion of male cases ( $90 \%$ in both), for which biometric measures are usually larger. ${ }^{40}$ Another important advantage of our study is the availability of longitudinal biometric measures from both second and third trimesters for a significant portion of our sample, thus allowing us to account for sampling bias 
medRxiv preprint doi: https://doi.org/10.1101/2020.08.09.20170811; this version posted August 23, 2020. The copyright holder for this preprint (which was not certified by peer review) is the author/funder, who has granted medRxiv a license to display the preprint in

All rights reserved. No reuse allowed without permission.

\section{Acknowledgements}

\section{Conclusions} ASD development.

between the two pregnancy periods and to investigate prenatal growth trajectories of these biometric measures in ASD and the two control groups. Using this data, we show that significant head growth abnormalities that are seen in mid-gestation (second trimester) for the ASD and TDS groups are attenuated towards the end of the pregnancy. While this attenuation could be due to accelerated growth in some fetuses like is seen for the TDS males, it could also be attributed to the typical higher variability of gestation-based biometric measures during the third trimester. ${ }^{48}$ Finally, this study is the first to examine correlations between prenatal US measures and ASD clinical metrics, allowing us to understand the associations between fetal growth and ASD severity.

Our study has several limitations. First, the sample size of the study, despite being the largest of its kind to date, is still too small to address associations within subgroups. This is particularly relevant to the longitudinal analyses (Figure $4 \&$ table 7) and to the correlations between the biometric measures and ASD symptoms (Figure 5) where data was available only for subsets of our sample. The same limitation applies to the sexspecific analyses since females comprised only $\sim 20 \%$ of the cases due to the inherent male bias of ASD. ${ }^{26}$ In addition, parents of children in the study sample were slightly younger than those of the other children with ASD in the NARC cohort. However, this small difference in parental age is unlikely to affect the study findings. Finally, we had only limited data about potential birth and pregnancy confounding factors for subjects in our study, such as weight at birth. Nonetheless, the comparison of cases to both TDS and TDP control groups probably minimized the effect of such confounders.

Our findings suggest that abnormal fetal head growth is a familial trait of ASD, which is modulated by sex and is associated with the severity of the disorder. Thus, it could serve as an early biomarker for ASD. Integration of our findings with fetal brain research will shed important light on neuroanatomical embryonic milestones in

This study was supported by a grant from the Israeli Science Foundation (527/15). 
medRxiv preprint doi: https://doi.org/10.1101/2020.08.09.20170811; this version posted August 23, 2020. The copyright holder for this preprint (which was not certified by peer review) is the author/funder, who has granted medRxiv a license to display the preprint in All rights reserved. No reuse allowed without permission.

1 This study was conducted as part of the requirements to obtain a degree in medicine from the Joyce \& Irving

2 Goldman Medical School, Faculty of Health Sciences, Ben-Gurion University of the Negev.

3 We thank Mrs. Inez Mureink and Prof. Norm O'Rourke for critical reviewing and editing of the manuscript.

\section{Conflict of Interest}

5 The authors report no biomedical financial interests or potential conflicts of interest. 
medRxiv preprint doi: https://doi.org/10.1101/2020.08.09.20170811; this version posted August 23, 2020. The copyright holder for this preprint (which was not certified by peer review) is the author/funder, who has granted medRxiv a license to display the preprint in All rights reserved. No reuse allowed without permission.

\section{References:}

1. Kim, Y. S. \& Leventhal, B. L. Genetic Epidemiology and Insights into Interactive Genetic and Environmental Effects in Autism Spectrum Disorders. Biol. Psychiatry 77, 66-74 (2015).

2. Lyall, K. et al. The Changing Epidemiology of Autism Spectrum Disorders. Annu. Rev. Public Health 38, 81-102 (2017).

3. Wang, C., Geng, H., Liu, W. \& Zhang, G. Prenatal, perinatal, and postnatal factors associated with autism. Medicine (Baltimore). 96, e6696 (2017).

4. Ornoy, A., Weinstein-Fudim, L. \& Ergaz, Z. Prenatal factors associated with autism spectrum disorder (ASD). Reprod. Toxicol. 56, 155-169 (2015).

5. Stoner, R. et al. Patches of Disorganization in the Neocortex of Children with Autism. N. Engl. J. Med. 370, 1209-1219 (2014).

6. Kemper, T. L. \& Bauman, M. Neuropathology of Infantile Autism. J. Neuropathol. Exp. Neurol. 57, 645-652 (1998).

7. Courchesne, E., Campbell, K. \& Solso, S. Brain growth across the life span in autism: Age-specific changes in anatomical pathology. Brain Res. 1380, 138-145 (2011).

8. Courchesne, E. et al. Neuron Number and Size in Prefrontal Cortex of Children With Autism. JAMA 306, 2001 (2011).

9. Casanova, M. F. et al. Minicolumnar abnormalities in autism. Acta Neuropathol. 112, 287-303 (2006).

10. Courchesne, E., Gazestani, V. H. \& Lewis, N. E. Prenatal Origins of ASD: The When, What, and How of ASD Development. Trends Neurosci. 43, 326-342 (2020).

11. Redcay, E. \& Courchesne, E. When Is the Brain Enlarged in Autism? A Meta-Analysis of All Brain Size Reports. Biol. Psychiatry 58, 1-9 (2005).

12. Courchesne, E. Evidence of Brain Overgrowth in the First Year of Life in Autism. JAMA 290, 337 (2003). 
medRxiv preprint doi: https://doi.org/10.1101/2020.08.09.20170811; this version posted August 23, 2020. The copyright holder for this preprint (which was not certified by peer review) is the author/funder, who has granted medRxiv a license to display the preprint in All rights reserved. No reuse allowed without permission.

13. Hazlett, H. C. et al. Magnetic Resonance Imaging and Head Circumference Study of Brain Size in Autism. Arch. Gen. Psychiatry 62, 1366 (2005).

14. Schumann, C. M. et al. Longitudinal Magnetic Resonance Imaging Study of Cortical Development through Early Childhood in Autism. J. Neurosci. 30, 4419-4427 (2010).

15. Hazlett, H. C. et al. Early brain development in infants at high risk for autism spectrum disorder. Nature 542, 348-351 (2017).

16. Piven, J., Arndt, S., Bailey, J. \& Andreasen, N. Regional Brain Enlargement in Autism: A Magnetic Resonance Imaging Study. J. Am. Acad. Child Adolesc. Psychiatry 35, 530-536 (1996).

17. Brambilla, P. et al. Brain anatomy and development in autism: review of structural MRI studies. Brain Res. Bull. 61, 557-69 (2003).

18. Surén, P. et al. Early Growth Patterns in Children with Autism. Epidemiology 24, 660-670 (2013).

19. Shen, M. D. et al. Extra-axial cerebrospinal fluid in high-risk and normal-risk children with autism aged 2-4 years: a case-control study. The Lancet Psychiatry 5, 895-904 (2018).

20. Sacco, R., Gabriele, S. \& Persico, A. M. Head circumference and brain size in autism spectrum disorder: A systematic review and meta-analysis. Psychiatry Res. - Neuroimaging 234, 239-251 (2015).

21. Deutsch, C. K. \& Joseph, R. M. Brief report: cognitive correlates of enlarged head circumference in children with autism. J. Autism Dev. Disord. 33, 209-15 (2003).

22. Bedford, S. A. et al. Large-scale analyses of the relationship between sex, age and intelligence quotient heterogeneity and cortical morphometry in autism spectrum disorder. Mol. Psychiatry 25, 614-628 (2020).

23. Lai, M. C., Lombardo, M. V., Auyeung, B., Chakrabarti, B. \& Baron-Cohen, S. Sex/Gender Differences and Autism: Setting the Scene for Future Research. Journal of the American Academy of Child and Adolescent Psychiatry (2015). doi:10.1016/j.jaac.2014.10.003

24. Mandy, W. et al. Sex differences in autism spectrum disorder: Evidence from a large sample of 
medRxiv preprint doi: https://doi.org/10.1101/2020.08.09.20170811; this version posted August 23, 2020. The copyright holder for this preprint (which was not certified by peer review) is the author/funder, who has granted medRxiv a license to display the preprint in All rights reserved. No reuse allowed without permission.

children and adolescents. J. Autism Dev. Disord. (2012). doi:10.1007/s10803-011-1356-0

25. Werling, D. M. \& Geschwind, D. H. Sex differences in autism spectrum disorders. Current Opinion in Neurology (2013). doi:10.1097/WCO.0b013e32835ee548

26. Battle, D. E. Diagnostic and Statistical Manual of Mental Disorders (DSM). CoDAS 25, 191-2 (2013).

27. Loomes, R., Hull, L. \& Mandy, W. P. L. What Is the Male-to-Female Ratio in Autism Spectrum Disorder? A Systematic Review and Meta-Analysis. Journal of the American Academy of Child and Adolescent Psychiatry (2017). doi:10.1016/j.jaac.2017.03.013

28. Bloss, C. S. \& Courchesne, E. MRI Neuroanatomy in Young Girls With Autism. J. Am. Acad. Child Adolesc. Psychiatry 46, 515-523 (2007).

29. Schumann, C. M., Barnes, C. C., Lord, C. \& Courchesne, E. Amygdala Enlargement in Toddlers with Autism Related to Severity of Social and Communication Impairments. Biol. Psychiatry 66, 942-949 (2009).

30. Ecker, C. et al. Association Between the Probability of Autism Spectrum Disorder and Normative SexRelated Phenotypic Diversity in Brain Structure. JAMA Psychiatry 74, 329 (2017).

31. Fulceri, F., Guzzetta, A., Athanasiadou, A., Iaconianni, L. \& Scattoni, M. L. Antenatal ultrasound value in risk calculation for Autism Spectrum Disorder: A systematic review to support future research. Neurosci. Biobehav. Rev. 92, 83-92 (2018).

32. Gamliel, M., Ebstein, R., Yirmiya, N. \& Mankuta, D. Minor Fetal Sonographic Findings in Autism Spectrum Disorder. Obstet. Gynecol. Surv. 67, 176-186 (2012).

33. Hobbs, K. et al. A Retrospective Fetal Ultrasound Study of Brain Size in Autism. Biol. Psychiatry 62, 1048-1055 (2007).

34. Bonnet-Brilhault, F. et al. Autism is a prenatal disorder: Evidence from late gestation brain overgrowth. Autism Res. 11, 1635-1642 (2018).

35. Whitehouse, A. J. O., Hickey, M., Stanley, F. J., Newnham, J. P. \& Pennell, C. E. Brief Report: A Preliminary Study of Fetal Head Circumference Growth in Autism Spectrum Disorder. J. Autism Dev. 
medRxiv preprint doi: https://doi.org/10.1101/2020.08.09.20170811; this version posted August 23, 2020. The copyright holder for this preprint (which was not certified by peer review) is the author/funder, who has granted medRxiv a license to display the preprint in All rights reserved. No reuse allowed without permission.

Disord. 41, 122-129 (2011).

36. Unwin, L. M. et al. A Prospective Ultrasound Study of Prenatal Growth in Infant Siblings of Children With Autism. Autism Res. 9, 210-216 (2016).

37. Blanken, L. M. E. et al. A prospective study of fetal head growth, autistic traits and autism spectrum disorder. Autism Res. 11, 602-612 (2018).

38. La Batide-Alanore, A. Familial Aggregation of Fetal Growth Restriction in a French Cohort of 7,822 Term Births between 1971 and 1985. Am. J. Epidemiol. 156, 180-187 (2002).

39. Sandin, S. et al. The Familial Risk of Autism. JAMA 311, 1770 (2014).

40. Schwärzler, P., Bland, J. M., Holden, D., Campbell, S. \& Ville, Y. Sex-specific antenatal reference growth charts for uncomplicated singleton pregnancies at 15-40 weeks of gestation. Ultrasound Obstet. Gynecol. 23, 23-29 (2004).

41. Lord, C. et al. The autism diagnostic observation schedule-generic: a standard measure of social and communication deficits associated with the spectrum of autism. J. Autism Dev. Disord. 30, 205-23 (2000).

42. Albers, C. A. \& Grieve, A. J. Test Review: Bayley, N. (2006). Bayley Scales of Infant and Toddler Development- Third Edition. San Antonio, TX: Harcourt Assessment. J. Psychoeduc. Assess. 25, 180190 (2007).

43. Warschausky, S. \& Raiford, S. E. Wechsler Preschool and Primary Scale of Intelligence. in Encyclopedia of Clinical Neuropsychology 3705-3709 (Springer International Publishing, 2018). doi:10.1007/978-3-319-57111-9_1606

44. Meiri, G. et al. Brief Report: The Negev Hospital-University-Based (HUB) Autism Database. $J$. Autism Dev. Disord. 47, 2918-2926 (2017).

45. Endres, L. K. \& Cohen, L. Reliability and validity of three-dimensional fetal brain volumes. $J$. Ultrasound Med. 20, 1265-1269 (2001).

46. Bartholomeusz, H. H., Courchesne, E. \& Karns, C. M. Relationship Between Head Circumference and 
medRxiv preprint doi: https://doi.org/10.1101/2020.08.09.20170811; this version posted August 23, 2020. The copyright holder for this preprint (which was not certified by peer review) is the author/funder, who has granted medRxiv a license to display the preprint in All rights reserved. No reuse allowed without permission.

Brain Volume in Healthy Normal Toddlers, Children, and Adults. Neuropediatrics 33, 239-241 (2002).

47. American Institute of Ultrasound in Medicine. AIUM Practice Guideline for the Performance of an Antepartum Obstetric Ultrasound Examination. J. Ultrasound Med. 22, 1116-1125 (2003).

48. Hadlock, F. P., Deter, R. L., Harrist, R. B. \& Park, S. K. Estimating fetal age: computer-assisted analysis of multiple fetal growth parameters. Radiology 152, 497-501 (1984).

49. Sarris, I. et al. Intra- and interobserver variability in fetal ultrasound measurements. Ultrasound Obstet. Gynecol. (2012). doi:10.1002/uog.10082

50. Perni, S. C. et al. Intraobserver and interobserver reproducibility of fetal biometry. Ultrasound Obstet. Gynecol. (2004). doi:10.1002/uog.1717

51. Hadlock, F., Deter, R., Harrist, R. \& Park, S. Fetal abdominal circumference as a predictor of menstrual age. Am. J. Roentgenol. 139, 367-370 (1982).

52. Hadlock, F. P., Deter, R. L., Harrist, R. B. \& Park, S. K. Fetal biparietal diameter: a critical reevaluation of the relation to menstrual age by means of real-time ultrasound. J. Ultrasound Med. 1,97104 (1982).

53. Hadlock, F., Deter, R., Harrist, R. \& Park, S. Fetal head circumference: relation to menstrual age. Am. J. Roentgenol. 138, 649-653 (1982).

54. Eklöf, E., Mårtensson, G. E., Ådén, U. \& Padilla, N. Reduced structural brain asymmetry during neonatal life is potentially related to autism spectrum disorders in children born extremely preterm. Autism Res. 12, 1334-1343 (2019).

55. Padilla, N. et al. Poor Brain Growth in Extremely Preterm Neonates Long Before the Onset of Autism Spectrum Disorder Symptoms. Cereb. Cortex bhv300 (2015). doi:10.1093/cercor/bhv300

56. Dinstein, I. \& Shelef, I. Anatomical brain abnormalities and early detection of autism. The Lancet Psychiatry 5, 857-859 (2018).

57. Hoekstra, R. A., Bartels, M., Verweij, C. J. H. \& Boomsma, D. I. Heritability of Autistic Traits in the 
medRxiv preprint doi: https://doi.org/10.1101/2020.08.09.20170811; this version posted August 23, 2020. The copyright holder for this preprint (which was not certified by peer review) is the author/funder, who has granted medRxiv a license to display the preprint in All rights reserved. No reuse allowed without permission.

General Population. Arch. Pediatr. Adolesc. Med. 161, 372 (2007).

58. Chaste, P. et al. Adjusting head circumference for covariates in autism: Clinical correlates of a highly heritable continuous trait. Biol. Psychiatry (2013). doi:10.1016/j.biopsych.2013.04.018

59. Wu, H. et al. Phenotype-to-genotype approach reveals head-circumference-associated genes in an autism spectrum disorder cohort. Clin. Genet. 97, 338-346 (2020).

60. Korevaar, T. I. M. et al. Association of maternal thyroid function during early pregnancy with offspring IQ and brain morphology in childhood: a population-based prospective cohort study. Lancet Diabetes Endocrinol. 4, 35-43 (2016).

61. Whitehouse, A. J. O. et al. Maternal Vitamin D Levels and the Autism Phenotype Among Offspring. J. Autism Dev. Disord. 43, 1495-1504 (2013).

62. Johnson, M. H., Jones, E. J. H. \& Gliga, T. Brain adaptation and alternative developmental trajectories. (2020). doi:10.1017/S0954579415000073 